Supporting Information

\title{
Nanosac, a Non-Cationic and Soft Polyphenol Nanocapsule, Enables Systemic Delivery of siRNA to Solid Tumors
}

Hyungjun Kim, Simseok A. Yuk, Alexandra M. Dieterly, Soonbum Kwon, Jinho Park,

Fanfei Meng, Hytham H. Gadalla, Maria Jose Cadena, L. Tiffany Lyle, Yoon Yeo

1 Supporting Table

31 Supporting Figures 
Supporting Table 1. Description of nanoparticles (NPs)

\begin{tabular}{|l|l|}
\hline NP & Description \\
\hline MSN & Mesoporous silica nanoparticles \\
\hline MSN $^{\mathrm{a}}$ & MSN conjugated with APTES \\
\hline MSN $^{\mathrm{a}}$-cy5 & Cy5-labeled MSN \\
\hline $\mathrm{MSN}^{\mathrm{a}} / \mathrm{siRNA}$ & MSN $^{\mathrm{a}}$ with siRNA loaded on the surface \\
\hline $\mathrm{MSN}^{\mathrm{a}} / \mathrm{pD}$ & MSN $^{\mathrm{a}}$ covered with pD \\
\hline $\mathrm{MSN}^{\mathrm{a}}$-cy5/pD & MSN $^{\mathrm{a}}$-cy5 covered with $\mathrm{pD}$ \\
\hline $\mathrm{MSN}^{\mathrm{a}} / \mathrm{siRNA} / \mathrm{pD}$ & $\mathrm{MSN}^{\mathrm{a}} /$ siRNA covered with $\mathrm{pD}$ \\
\hline $\mathrm{O} / \mathrm{pD}^{*}$ & Nanocapsules after MSN core removal \\
\hline $\mathrm{O}-\mathrm{cy} 5 / \mathrm{pD}$ & Cy5-labeled nanocapsules after MSN core removal \\
\hline $\mathrm{O} / \mathrm{siRNA} / \mathrm{pD}$ & siRNA-loaded nanocapsules after MSN core removal \\
\hline
\end{tabular}

*: Collectively called Nanosac. 

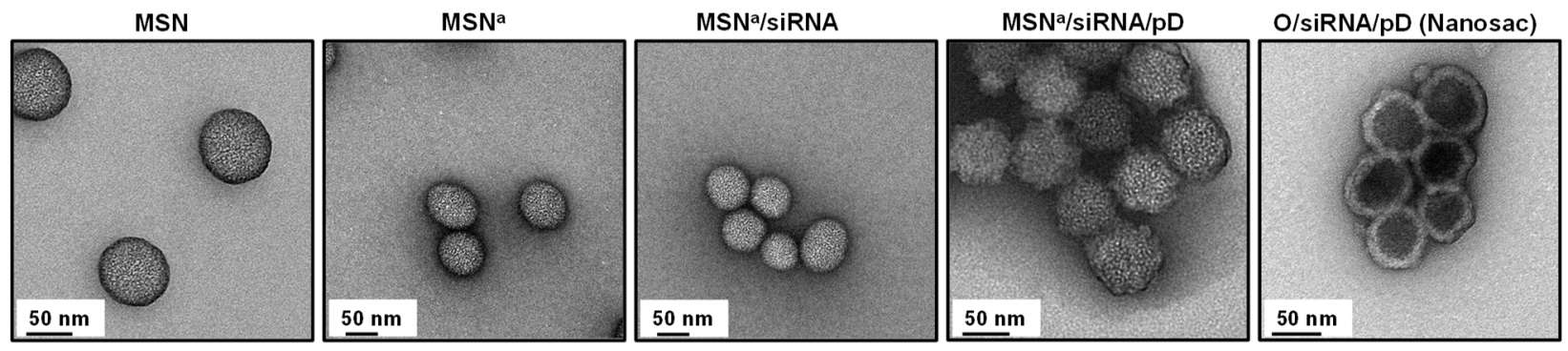

Supporting Fig. 1. TEM images of Nanosac and the precursors. Negatively stained with $1 \%$ phosphotungstic acid. Scale bars: $50 \mathrm{~nm}$. 

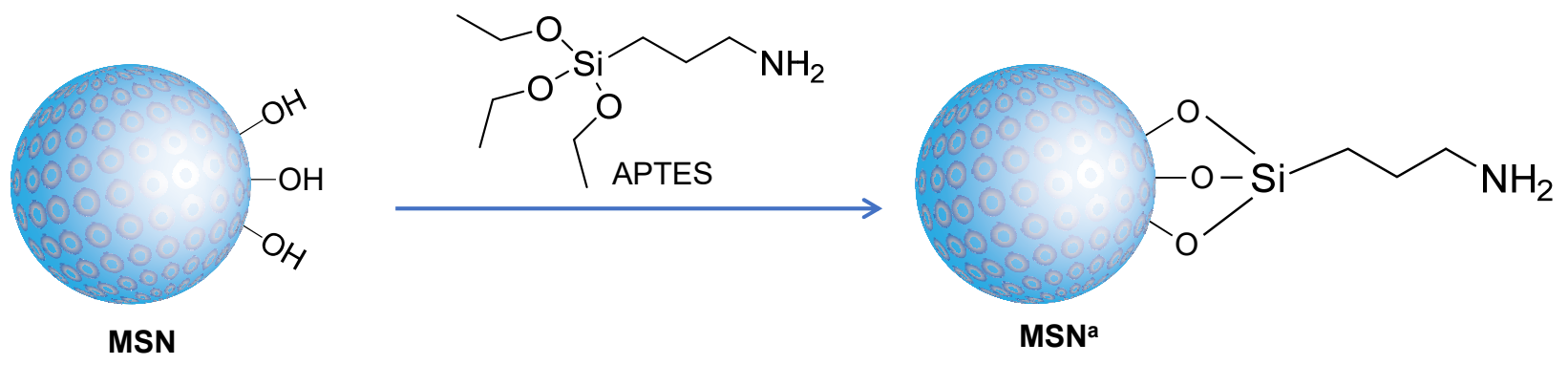

Supporting Fig. 2. Functionalization scheme of MSN to MSN ${ }^{a}$ [MSN conjugated with APTES (3-aminopropyltriethoxysilane)]. 


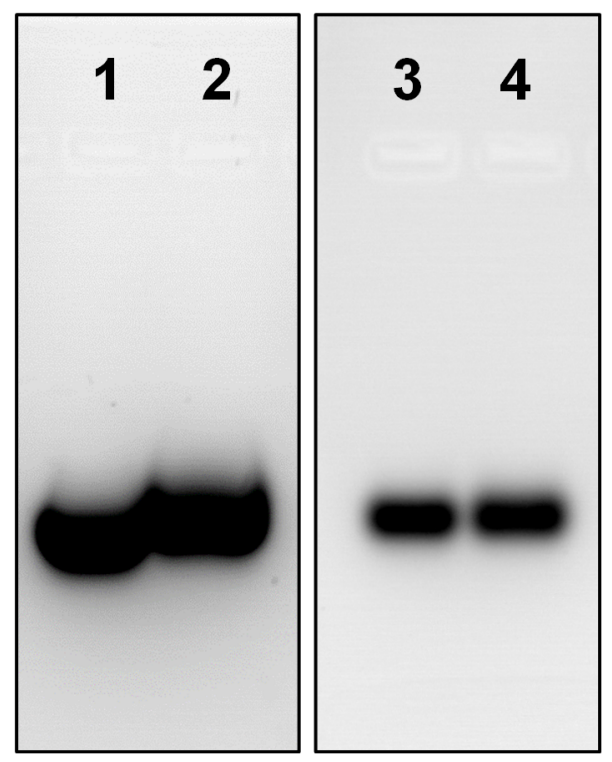

Supporting Fig. 3. Gel electrophoresis of siLuc and siLuc incubated in $2 \mathrm{M} \mathrm{HF} / 8 \mathrm{M} \mathrm{NH} 4 \mathrm{~F}$ for 5 min. Lane 1: siLuc $(100 \mu \mathrm{M})$, Lane 2: siLuc $+2 \mathrm{M} \mathrm{HF} / 8 \mathrm{M} \mathrm{NH} 4 \mathrm{~F}($ siLuc: $100 \mu \mathrm{M})$, Lane 3: Lane $1 \times 1 / 10$ (siLuc: $10 \mu \mathrm{M}$ ), Lane 4: Lane $2 \times 1 / 10$ (siLuc: $10 \mu \mathrm{M}$ ). 

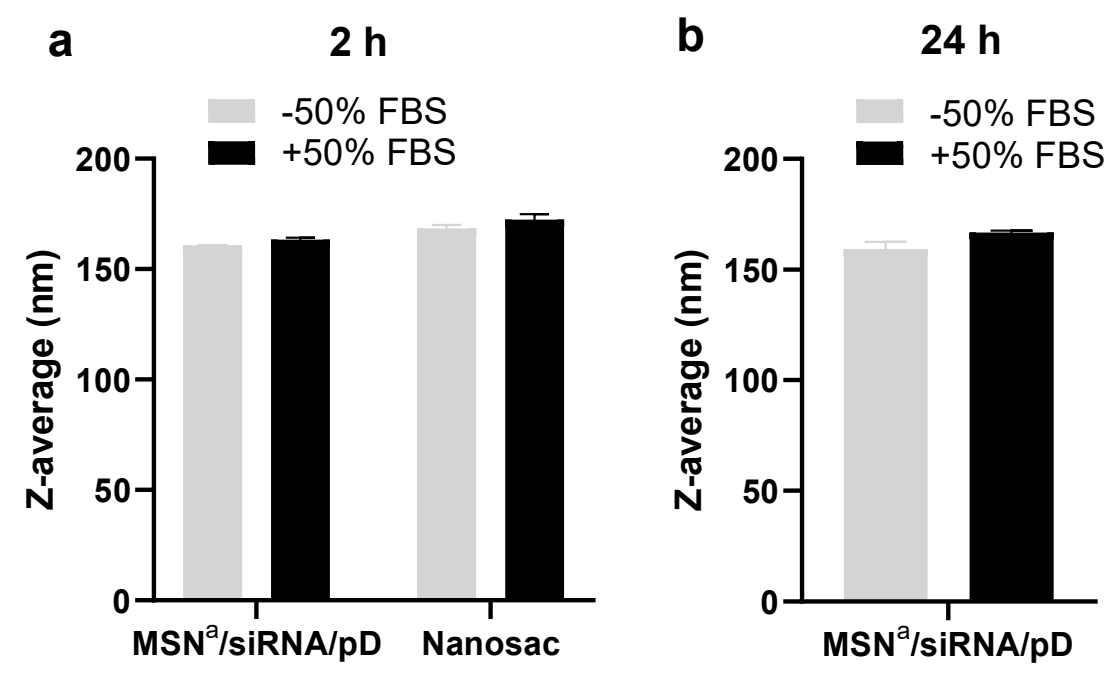

Supporting Fig. 4. Size distribution of (a) MSNa/siRNA/pD and Nanosac before (-) and after (+) incubation in $50 \%$ FBS for $2 \mathrm{~h}$ and (b) $\mathrm{MSN}^{\mathrm{a}} / \mathrm{siRNA} / \mathrm{pD}$ before $(-)$ and after $(+)$ incubation in $50 \%$ FBS for $24 \mathrm{~h}$ at $37^{\circ} \mathrm{C}$. $(\mathrm{n}=3$ tests of a representative batch, mean \pm s.d.) 

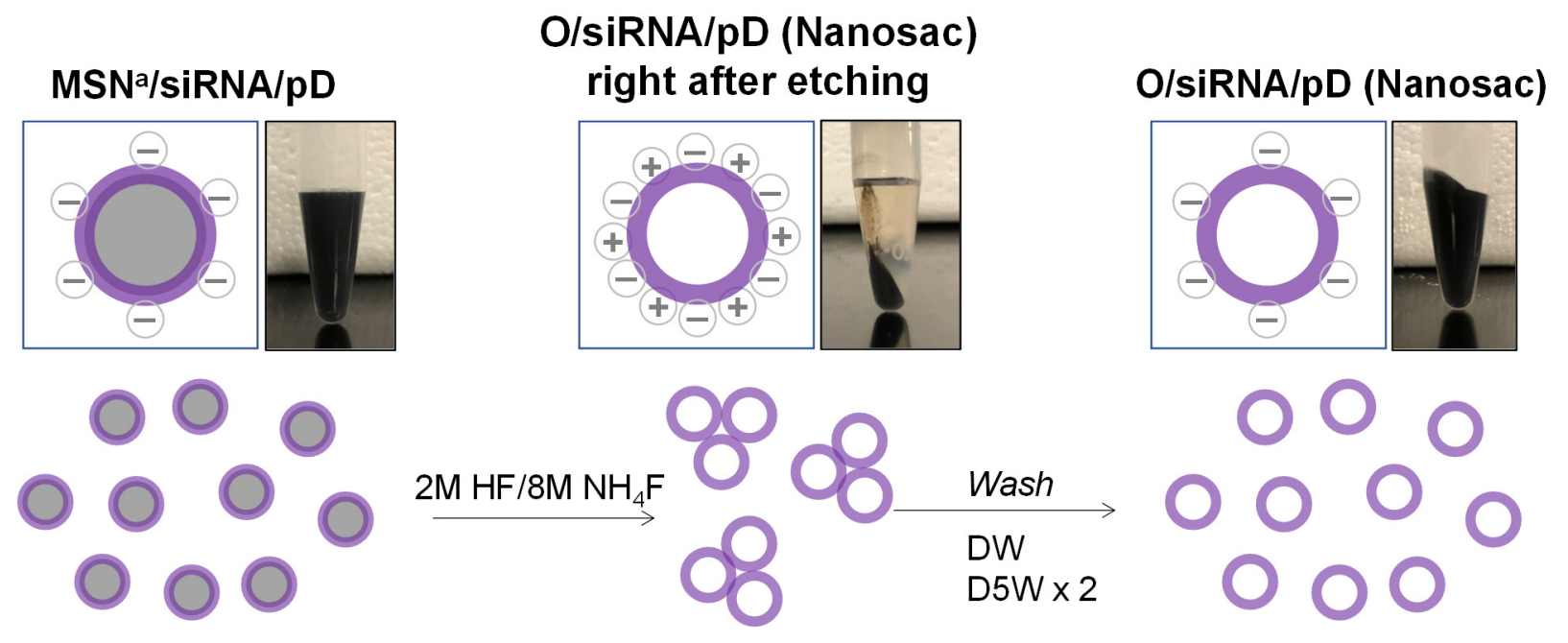

Supporting Fig. 5. Schematic overview of flocculation and deflocculation of $\mathrm{O} / \mathrm{siRNA} / \mathrm{pD}$ (Nanosac). Flocculation occurs with the addition of etching solution $(2 \mathrm{M} \mathrm{HF} / 8 \mathrm{M} \mathrm{NH} 4 \mathrm{~F})$ to $\mathrm{MSN}^{\mathrm{a}} / \mathrm{siRNA} / \mathrm{pD}$. Nanosac is deflocculated after repeated rinsing with DW and D5W accompanied by gentle sonication. 
a

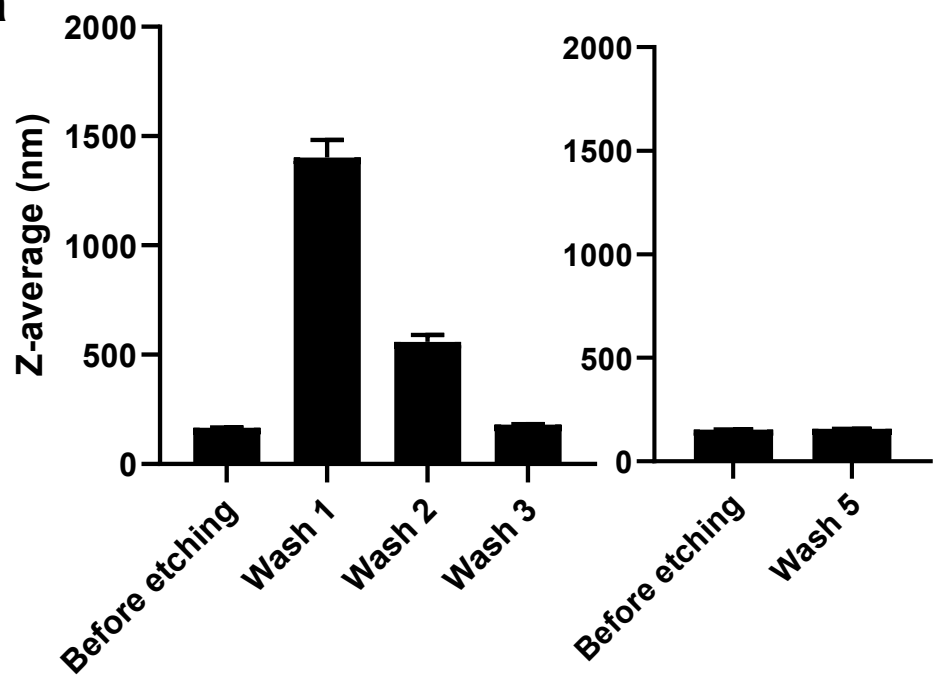

b

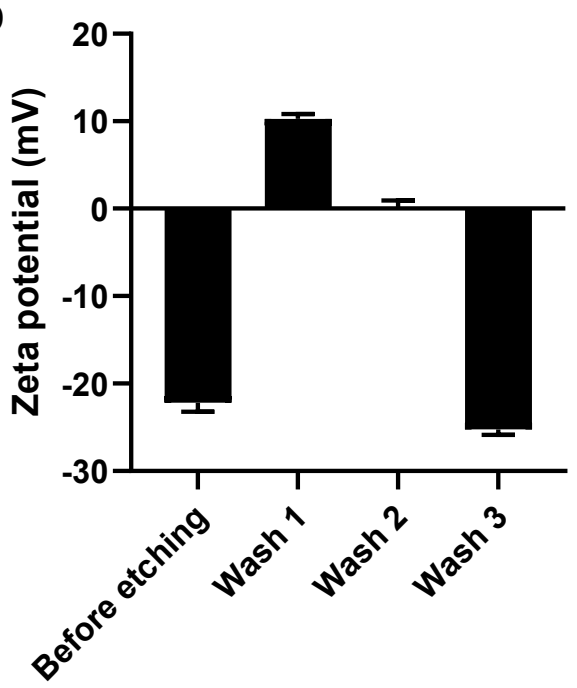

Supporting Fig. 6. (a) Z-average and (b) zeta potential of NPs through core etching and washing processes. 

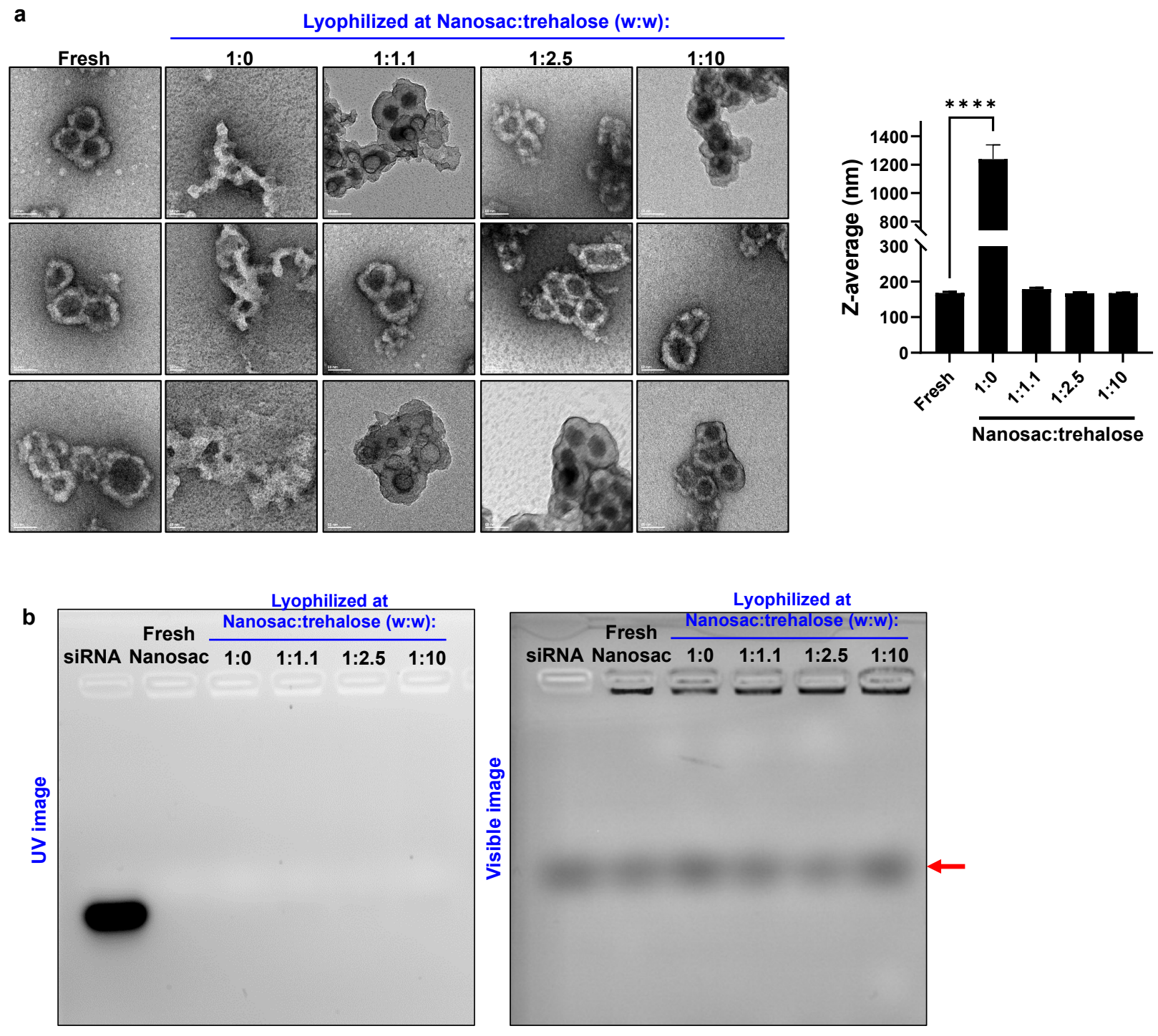

Supporting Fig. 7. (a) TEM images and particle sizes of Nanosac lyophilized in varying Nanosac:trehalose (w:w) ratios. (b) Gel electrophoresis of Nanosac, fresh or lyophilized with trehalose, demonstrating the stability of siRNA encapsulation. The diffuse bands indicated by the red arrow in the visible image panel correspond to the gel loading dye. 

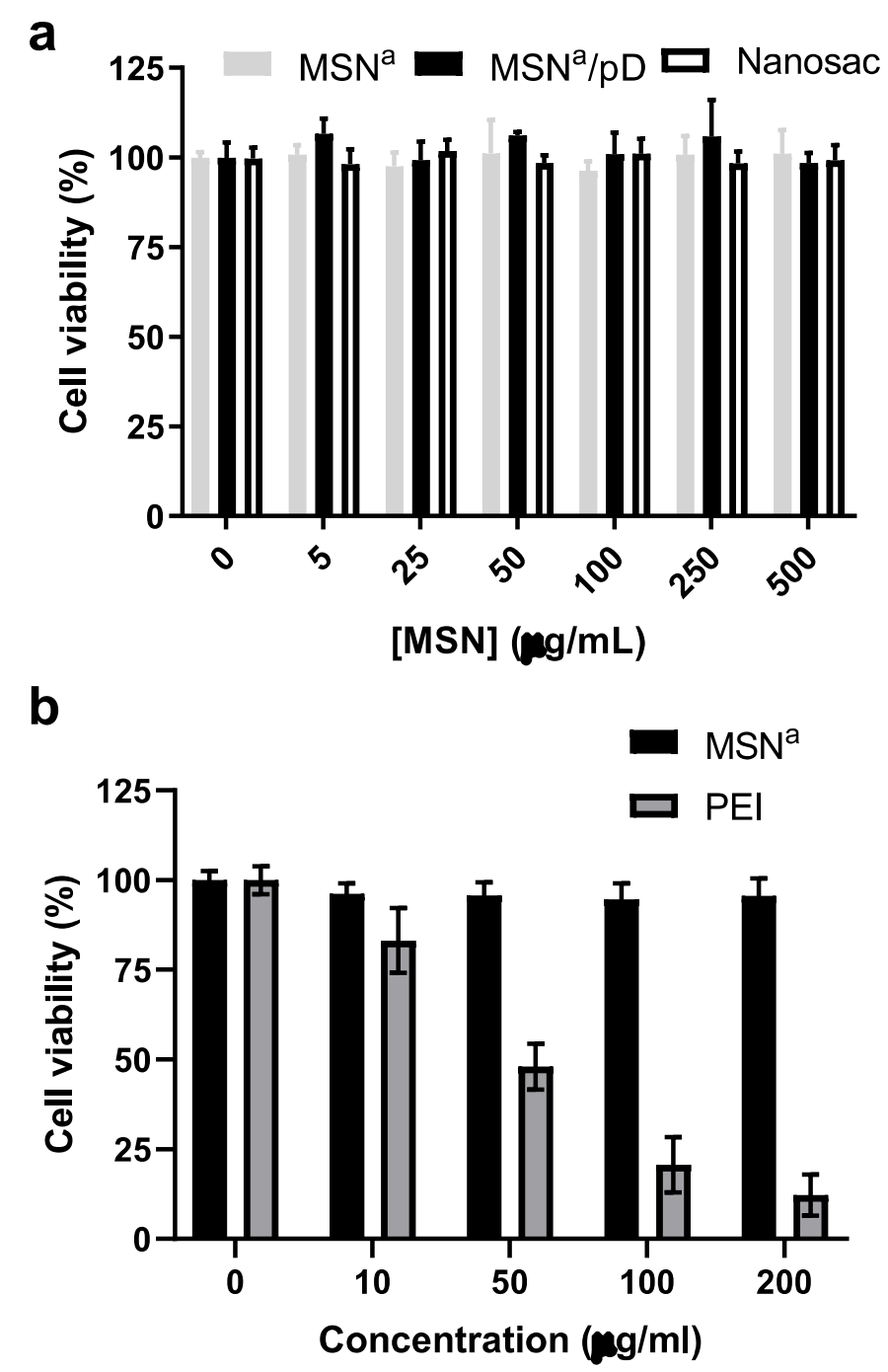

Supporting Fig. 8. (a) Cytotoxicity of $\mathrm{MSN}^{\mathrm{a}}, \mathrm{MSN} / \mathrm{pD}$, and Nanosac on CT26 cells. (b) Cytotoxicity of $\mathrm{MSN}^{\mathrm{a}}$ compared with polyethyleneimine (PEI) ( $\mathrm{n}=3$ tests of a representative batch, mean \pm s.d.). Two-way ANOVA followed by Dunnett's multiple comparisons test. At all concentrations, NPs showed no difference from non-treated control. 


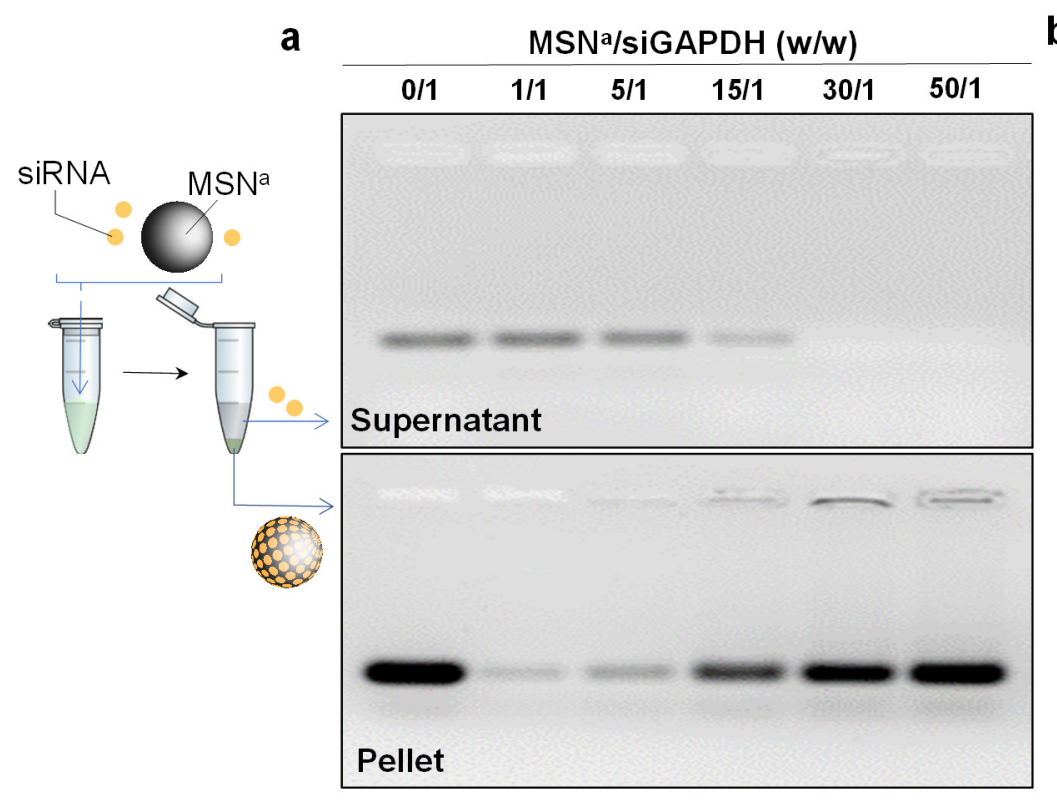

b $\frac{\text { MSNa/siLuc }}{0 / 1200 / 1100 / 1}$

C MSNa/SiPD-L1

$0 / 1200 / 1$ 100/1 0/1 200/1 100/1
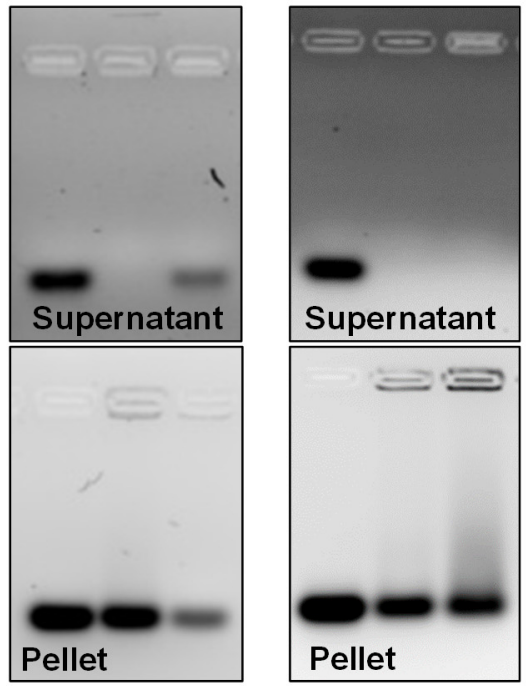

Supporting Fig. 9. Gel electrophoresis of supernatants and pellets of MSN $/$ siRNA (siGAPDH, siLuc, or siPD-L1) complexes at various weight ratios of $\mathrm{MSN}^{\mathrm{a}}$ to siRNA. 0/1 indicates siRNA only: "pellet" and "supernatant" appear in different intensities in (a), where "pellet" shows 10 $\mu \mathrm{M}$ of siRNA, whereas "supernatant" shows $10 \mu \mathrm{M}$ of siRNA diluted in the $100 \mu \mathrm{L}$ of HEPES buffer. 


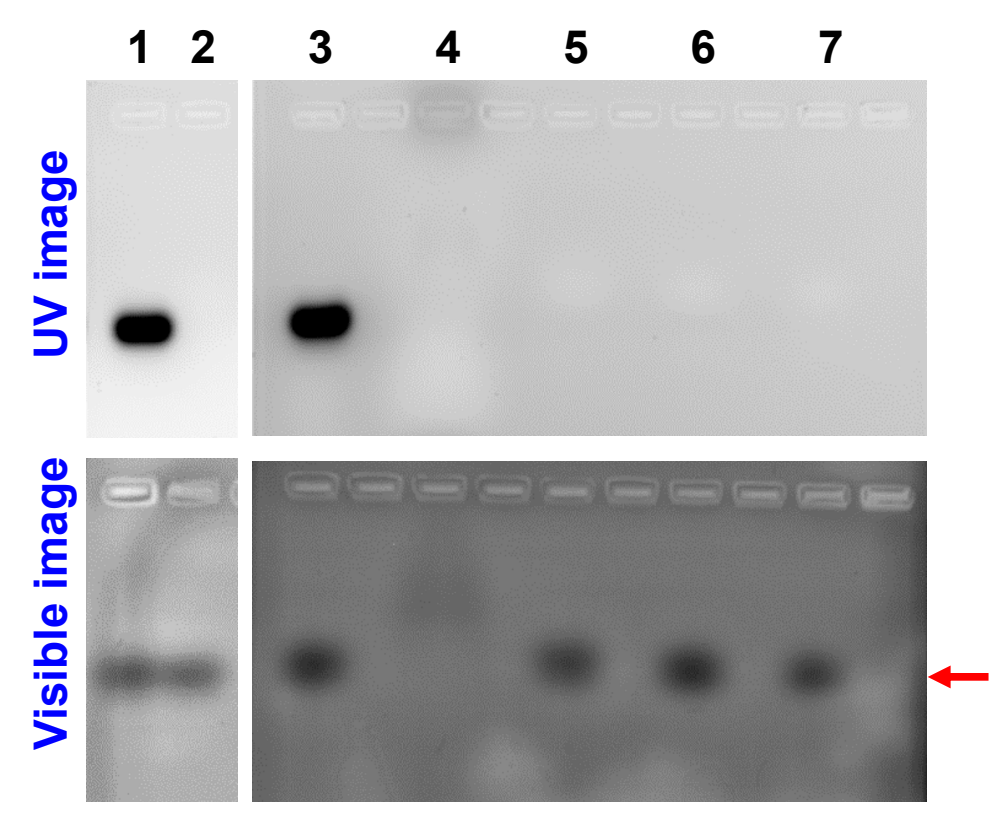

Supporting Fig. 10. UV and visible image of gel electrophoresis of waste solution from each production step. Lane 1: control siRNA; Lane 2: dopamine solution; Lane 3: control siRNA; Lane 4: etching solution; Lane 5: $1^{\text {st }}$ wash; Lane 6: $2^{\text {nd }}$ wash; Lane 7: $3^{\text {rd }}$ wash. The diffuse bands indicated by the red arrow in the visible image panel correspond to the gel loading dye. 
a

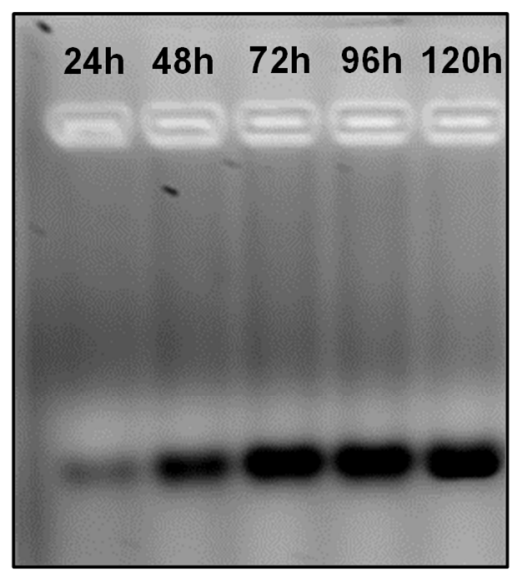

b

$\ln \mathrm{DW}(\mathrm{pH} 3)$

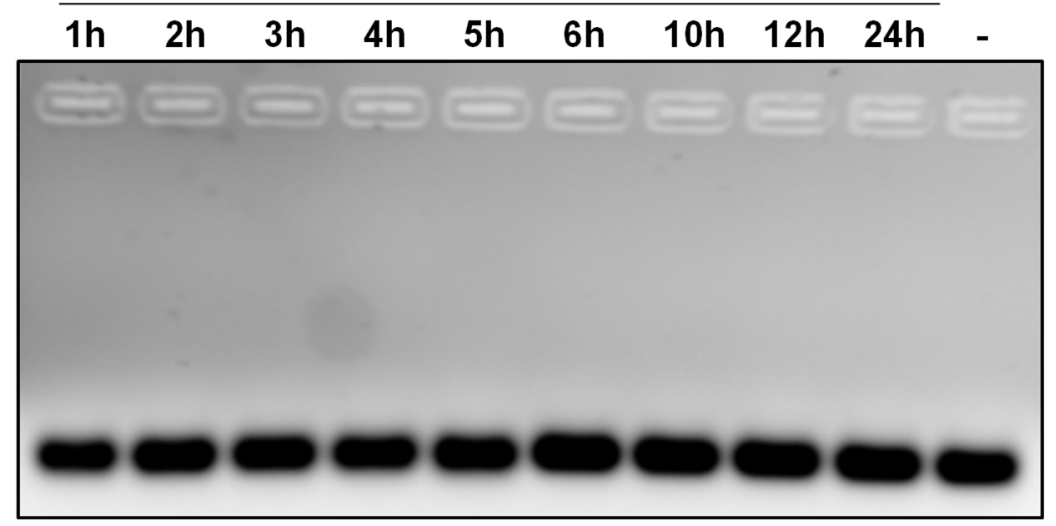

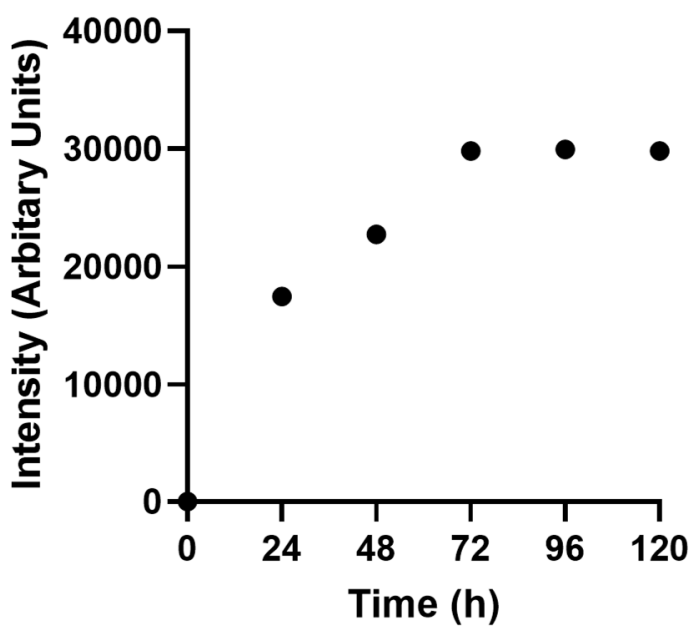

Time (h)

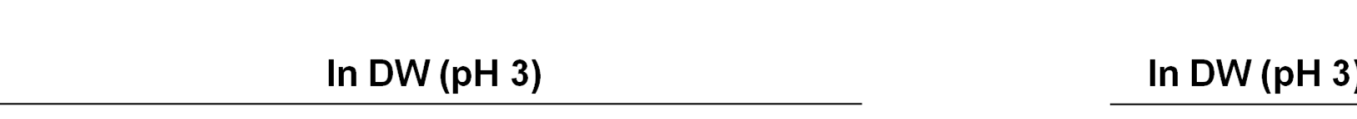

- 24h 48h 72h

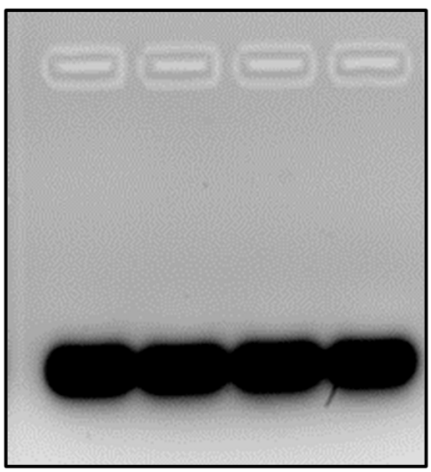

Supporting Fig. 11. (a) Gel electrophoresis and quantitative presentation of siRNA released from Nanosac in DW (pH 3) in $120 \mathrm{~h}$. (b) Stability of siRNA incubated in DW (pH 3) for $24 \mathrm{~h}$ or $72 \mathrm{~h}$. 


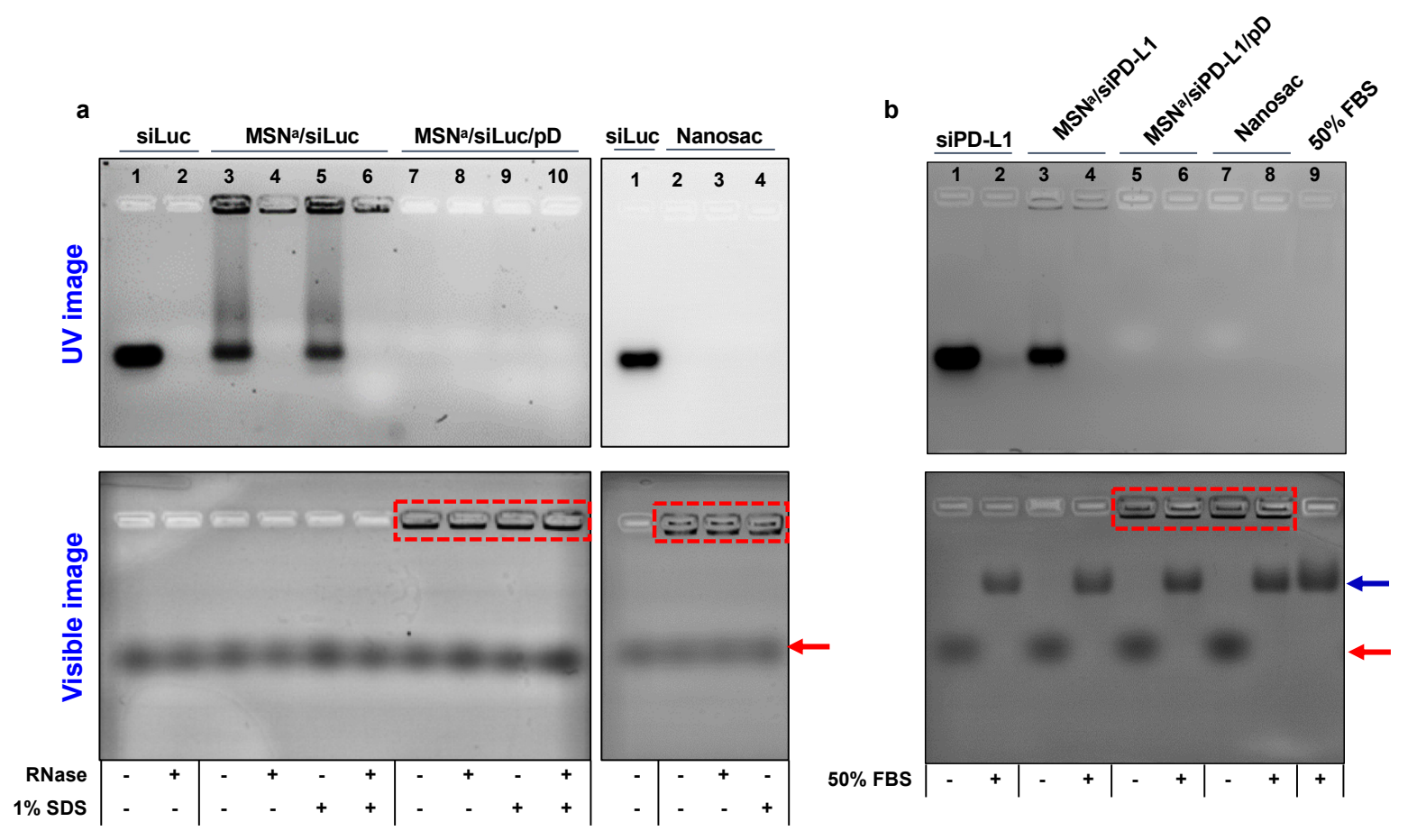

Supporting Fig. 12. UV and visible image of gel electrophoresis of (a) siLuc, MSNª/siLuc, $\mathrm{MSN}^{\mathrm{a}} / \mathrm{siLuc} / \mathrm{pD}$, and Nanosac with/without RNase \pm SDS challenge and (b) siPD-L1, $\mathrm{MSN}^{\mathrm{a}} / \mathrm{siPD}-\mathrm{L} 1, \mathrm{MSN} / \mathrm{siPD}-\mathrm{L} 1 / \mathrm{pD}$, and Nanosac with/without 50\% FBS challenge. siRNA or NPs were challenged with $166 \mathrm{U} / \mathrm{mL}$ RNase for $15 \mathrm{~min} \pm 8 \mathrm{mg} / \mathrm{mL}$ SDS for additional $2.5 \mathrm{~h}$ or $50 \%$ FBS for $1 \mathrm{~h}$, both at $37^{\circ} \mathrm{C}$, and analyzed by agarose gel electrophoresis. Dark signals in dashed boxes of visible images indicate $\mathrm{MSN}^{\mathrm{a}} / \mathrm{siRNA} / \mathrm{pD}$ and Nanosac stuck in the wells, not releasing and subjecting the siRNA to degradation by the challenge conditions. The diffuse bands indicated by the red arrow in the visible image panel correspond to the gel loading dye. The bands indicated by the blue arrow in the visible image show only in samples containing FBS (corresponding disappearance of bands indicated by the red arrow), likely due to dye complexation with proteins in FBS. 


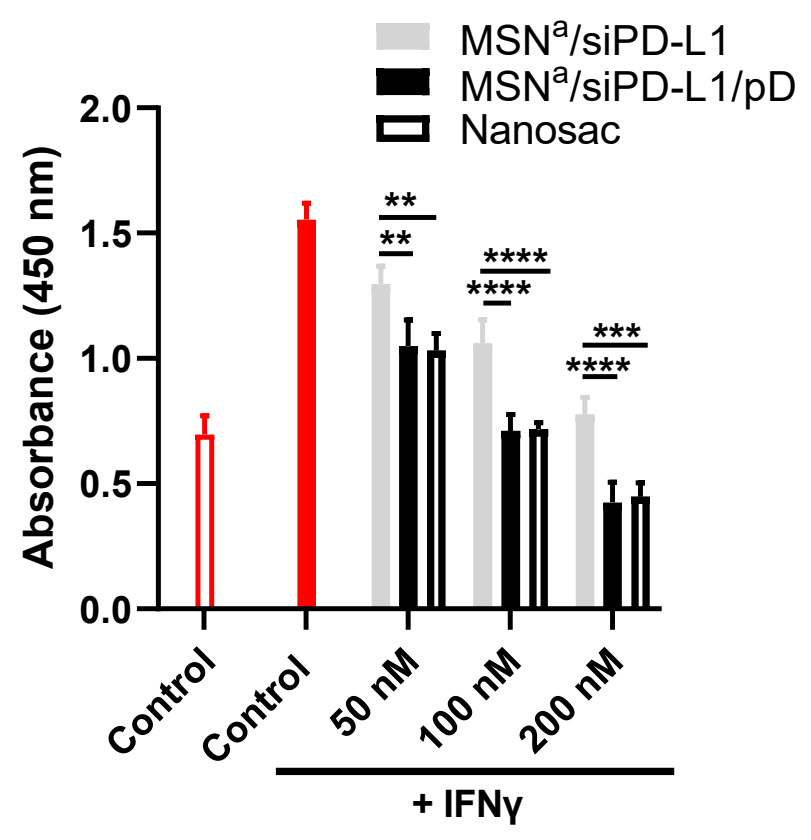

Supporting Fig. 13. Silencing of PD-L1 expression in IFN- $\gamma$-activated CT2 6 cells by $\mathrm{MSN}^{\mathrm{a}} / \mathrm{siPD}-\mathrm{L} 1, \mathrm{MSN} / \mathrm{siPD}-\mathrm{L} 1 / \mathrm{pD}$, and Nanosac, measured after $48 \mathrm{~h}$ treatment in complete medium. siPD-L1 concentration varied from $50 \mathrm{nM}$ to $200 \mathrm{nM}$. ( $\mathrm{n}=3$ test of a representative batch, mean \pm s.d.). ${ }^{* *}: \mathrm{p}<0.01 ; * * *$ p $<0.001,{ }^{* * * *}: \mathrm{p}<0.0001$ by Tukey's multiple comparisons test following two-way ANOVA. 

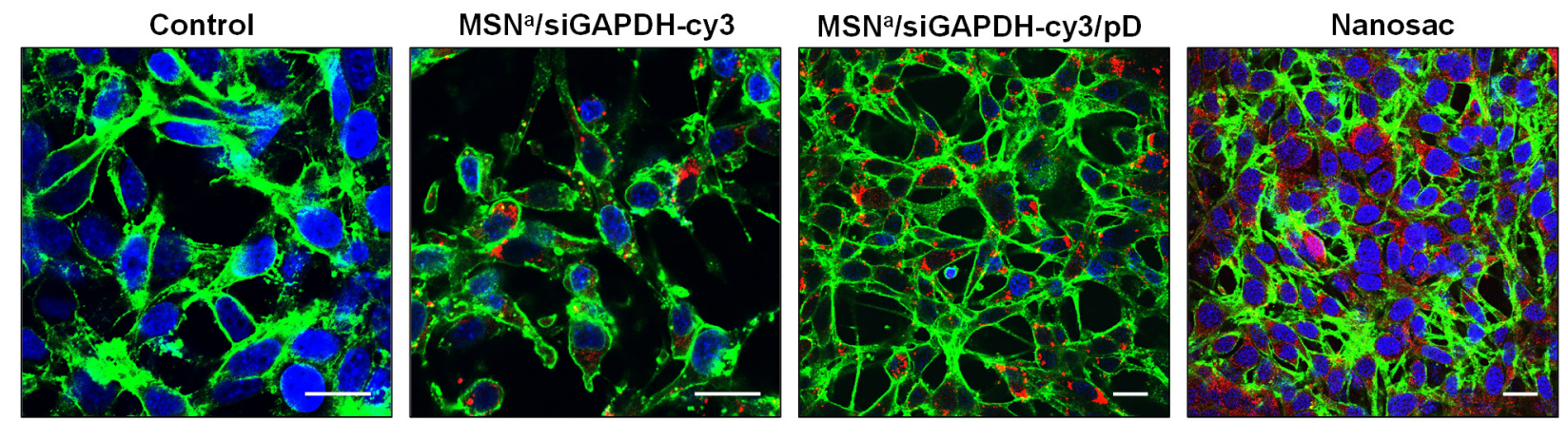

Cell membrane; Nuclei; siGAPDH-cy3

Supporting Fig. 14. Confocal microscope images of CT26 cells incubated with MSN siGAPDH-cy3, MSNa/siGAPDH-cy3/pD, and Nanosac. Green: Wheat Germ Agglutinin (cell membrane); Red: siGAPDH-cy3; Blue: Hoechst 33342 (nuclei). Scale bars: $20 \mu \mathrm{m}$. 


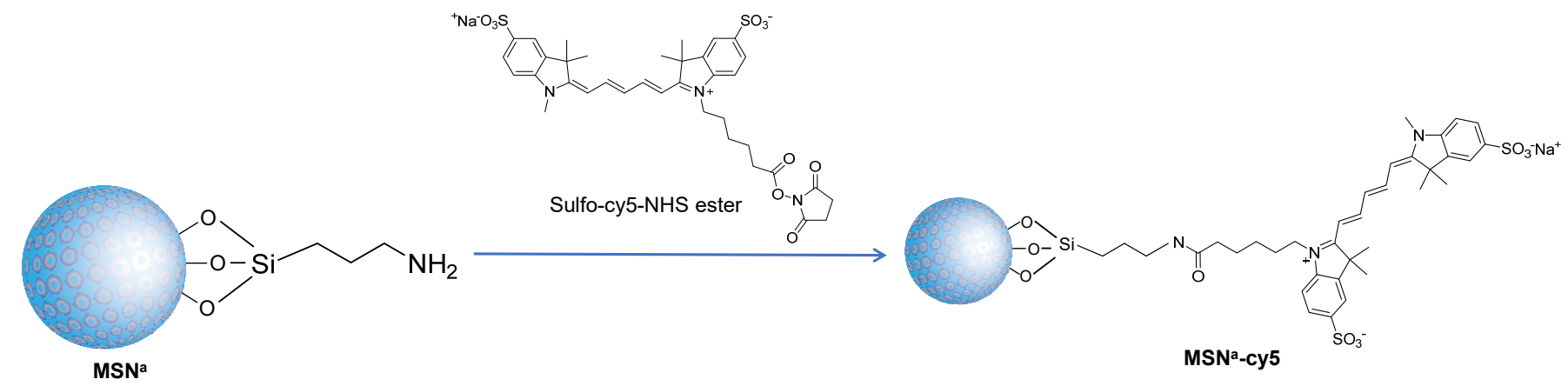

Supporting Fig. 15. Synthesis schematic of cy5-labeled MSNª (MSN-cy5). 


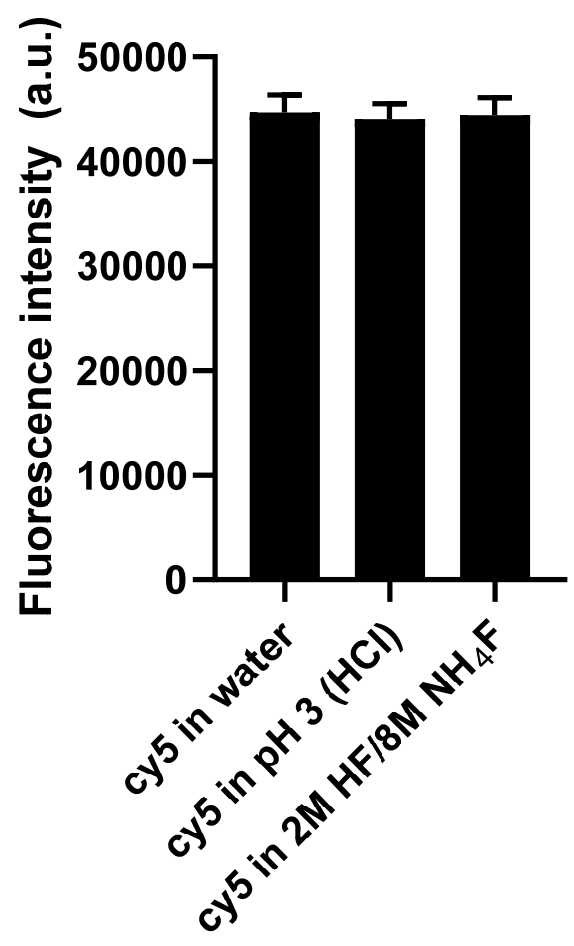

Supporting Fig. 16. Fluorescence intensity of cy5 after incubation in DW, pH 3, and 2M HF/8M $\mathrm{NH}_{4} \mathrm{~F}$ for 5 min. $\mathrm{n}=3$ tests of a representative batch (mean \pm s.d). 


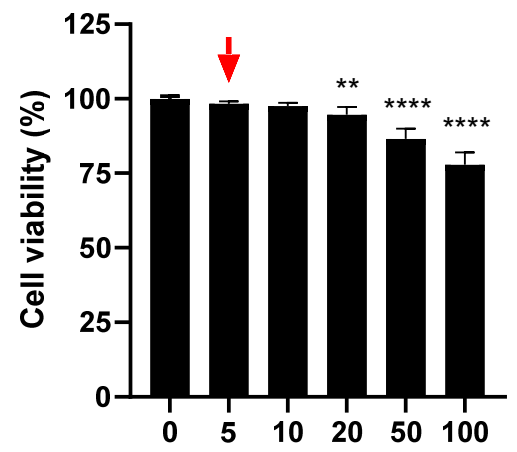

[Chlorpromazine] $(\mu \mathrm{M})$
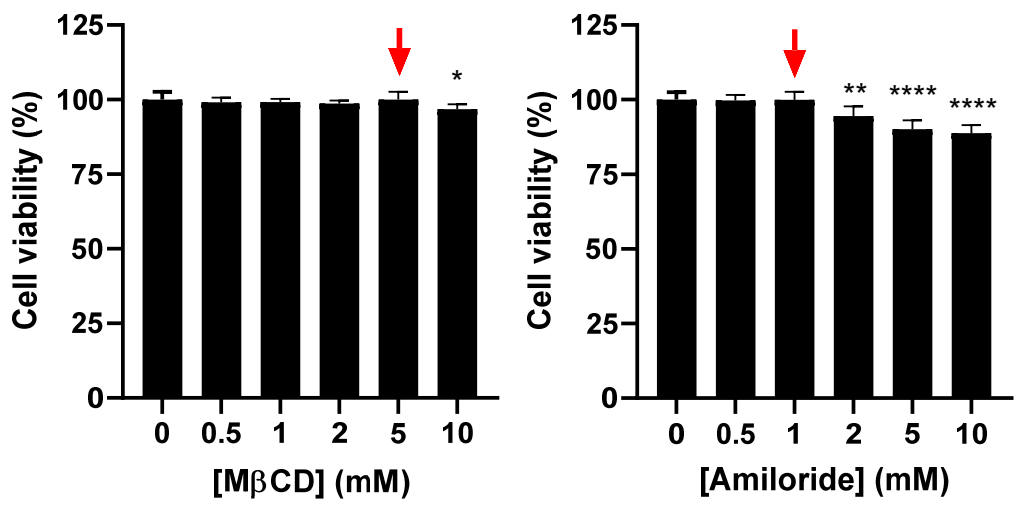

Supporting Fig. 17. Cytotoxicity of chlorpromazine, methyl- $\beta$-cyclodextrin, and amiloride on CT26 cells. CT26 cells were incubated with each inhibitor for $30 \mathrm{~min} . \mathrm{n}=6$ tests of a representative batch, mean \pm s.d. *: $\mathrm{p}<0.05 ;{ }^{*}: \mathrm{p}<0.01 ; * * *: \mathrm{p}<0.001 ; * * * *: \mathrm{p}<0.0001$ vs 0 in each treatment by Dunnett's multiple comparisons test following two-way ANOVA. Arrows indicate the concentration used in the endocytosis inhibition study (Fig. 3a). 


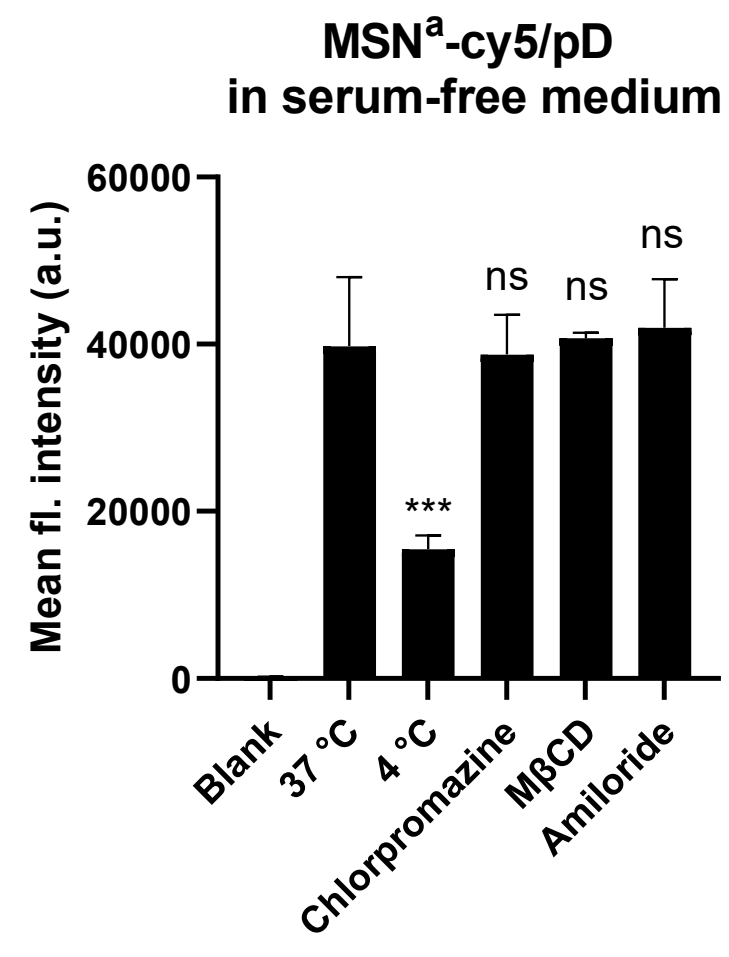

Supporting Fig. 18. Effects of endocytosis inhibitors on NP uptake in serum-free medium. CT26 cells were preincubated for 30 min with each inhibitor at a subtoxic concentration, followed by treatment of $\mathrm{MSN}^{\mathrm{a}}$-cy5/pD in serum-free medium for $2 \mathrm{~h}(\mathrm{n}=3$ tests of a representative batch, mean \pm s.d.). ${ }^{* * *}: \mathrm{p}<0.001$ vs $37^{\circ} \mathrm{C}$ by Dunnett's multiple comparisons test following one-way ANOVA. 

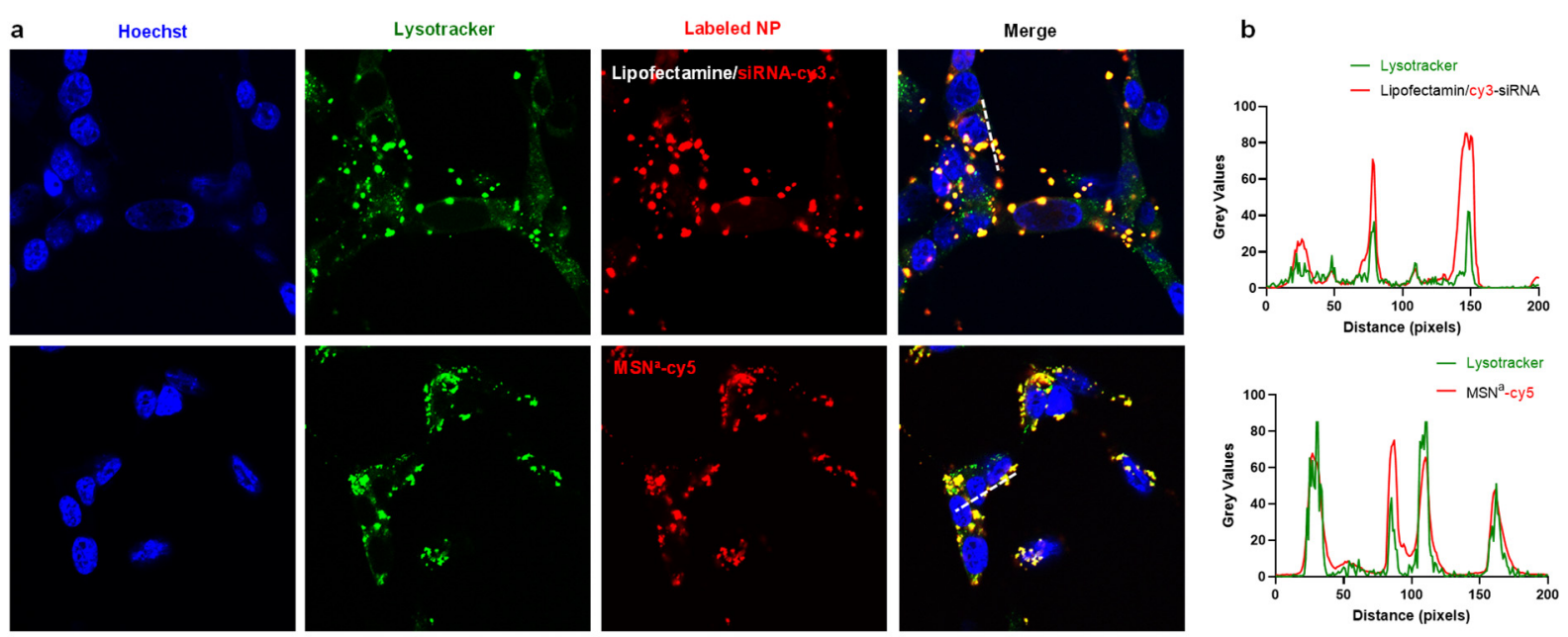

C
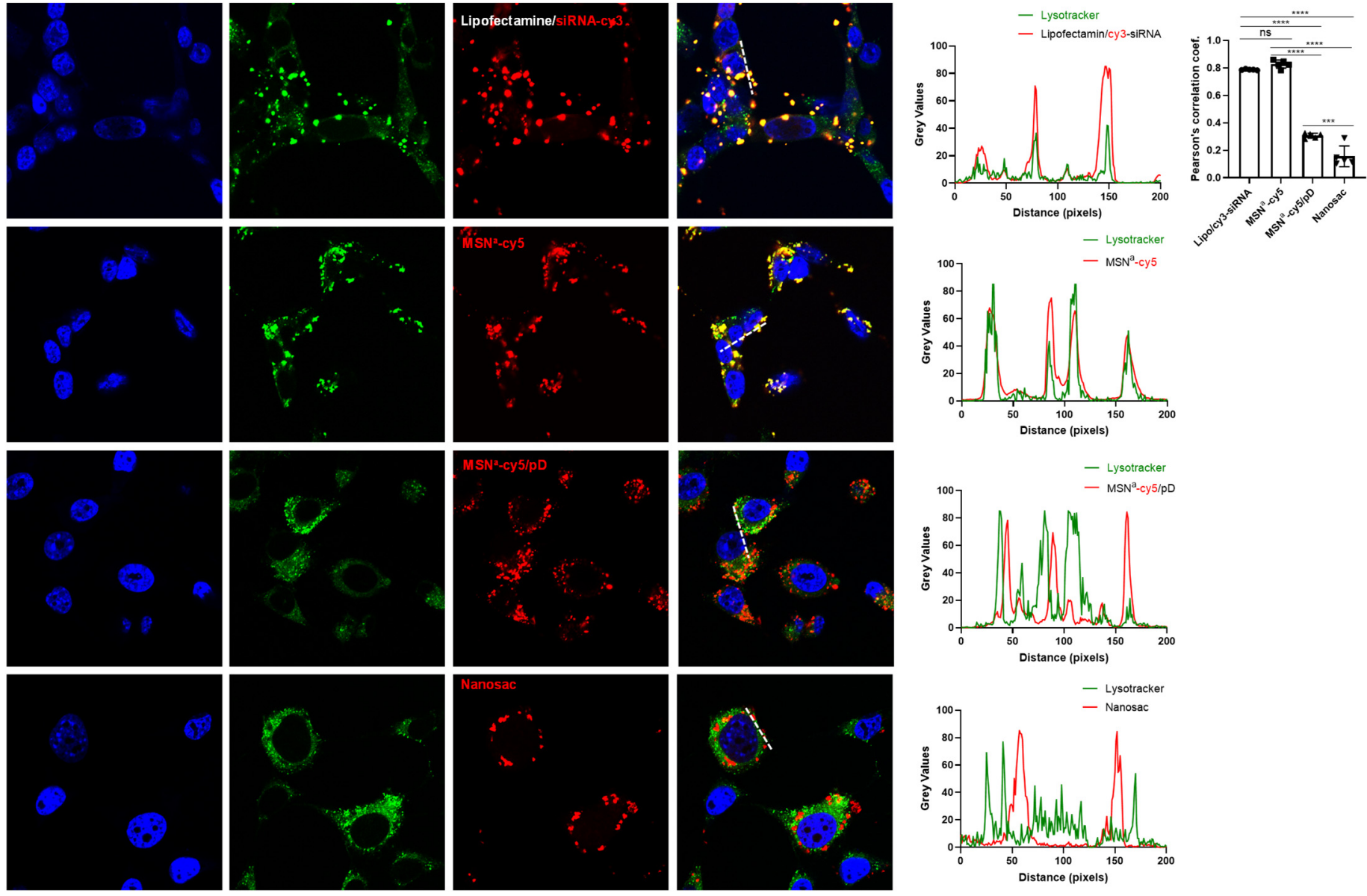

Supporting Fig. 19. (a) Intracellular trafficking of Lipofectamine/siRNA-cy3, cy5-labeled $\mathrm{MSN}^{\mathrm{a}}, \mathrm{MSN}$ / $/ \mathrm{pD}$, and Nanosac in CT26 cells; (b) Fluorescence intensity profiles along the white lines in (a); (c) Pearson's correlation coefficients indicating the degree of NP/lysosome colocalization in confocal images: $\mathrm{R}=1$ (perfect colocalization), $\mathrm{R}=0$ (no colocalization). $\mathrm{n}=5$ tests of a representative batch (mean $\pm \mathrm{s.d}$ ). ${ }^{* * *}: \mathrm{p}<0.001$; $* * * *: \mathrm{p}<0.0001$; ns: not significant by Tukey's multiple comparisons test following one-way ANOVA. Green: Lysotracker (lysosome); Red: cy5-labeled NPs; Blue: Hoechst 33342 (nuclei). 


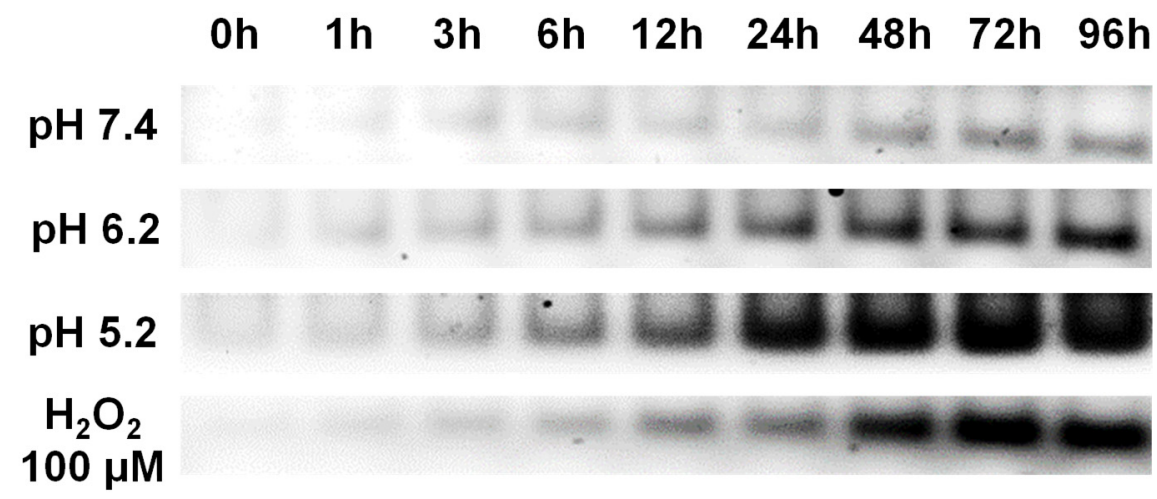

Supporting Fig. 20. Gel electrophoresis of siRNA release kinetics from Nanosac, performed in different $\mathrm{pH}$ and $\mathrm{H}_{2} \mathrm{O}_{2}(100 \mu \mathrm{M})$ with constant agitation at $37^{\circ} \mathrm{C}$. A representative image of 3 replicates. 

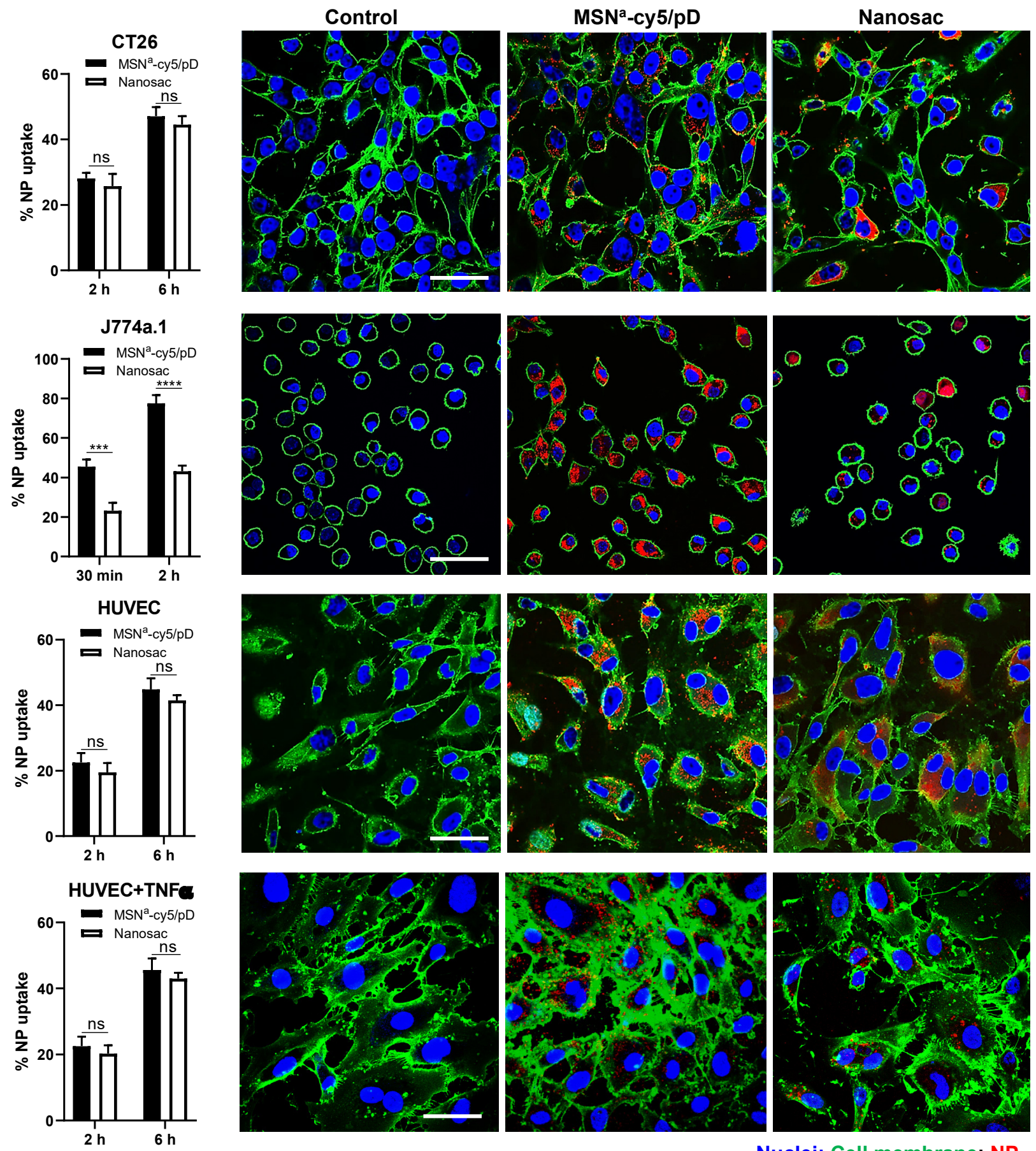

Nuclei; Cell membrane; NP

Supporting Fig. 21. Quantitative measurement and confocal microscope images of CT26, J774a.1, HUVEC, and TNF- $\alpha$ activated HUVEC cells incubated with $\mathrm{MSN}^{\mathrm{a}}$-cy5/pD and Nanosac in. $\mathrm{n}=3$ tests of a representative batch (mean $\pm \mathrm{s} . \mathrm{d}) . * * *: \mathrm{p}<0.001$ and $* * * *: \mathrm{p}<$ 0.0001 by Sidak's multiple comparisons test following two-way ANOVA. Green: Wheat Germ Agglutinin (cell membrane); Red: cy5-labeled NPs; Blue: Hoechst 33342 (nuclei). Scale bars: 50 $\mu \mathrm{m}$. 
a

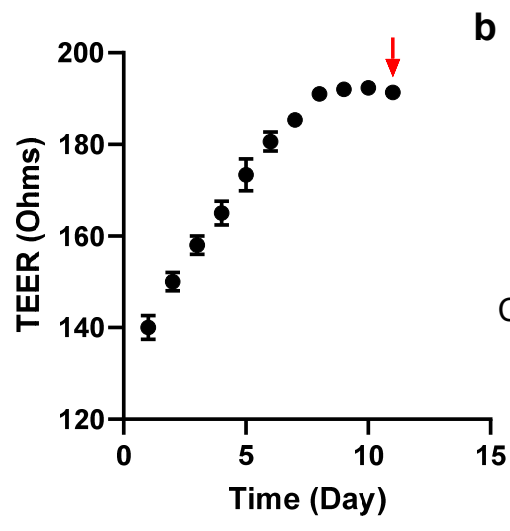

b

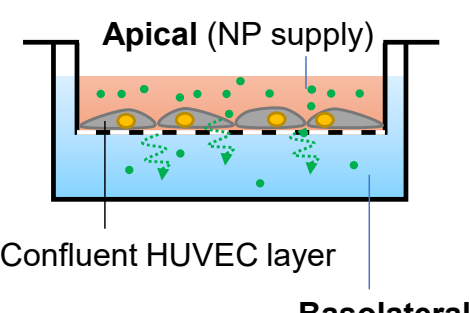

Basolateral

(NPs penetrating through HUVEC layer)

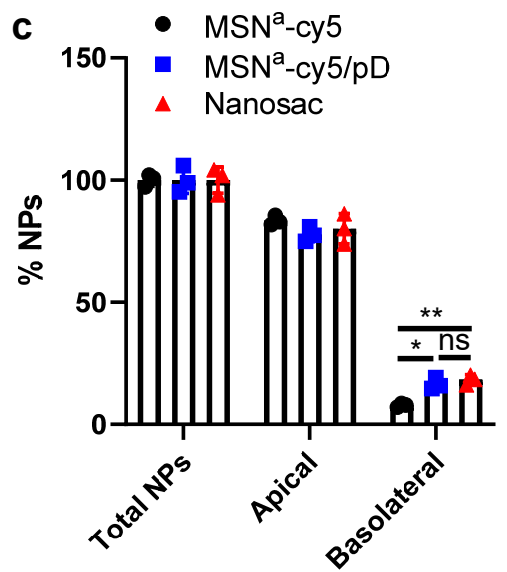

Supporting Fig. 22. (a) Representative Trans-Epithelial Electrical Resistance (TEER) of HUVEC monolayer $(n=3$, mean \pm s.d.). The arrow indicates the time point NPs were added to the HUVEC layer. (b) Schematic of transendothelial transport of NPs. (c) The percentage of NP transport across the TNF- $\alpha$-activated HUVEC monolayer $(n=3$ tests of a representative batch, mean \pm s.d.). $*: p<0.05$ and $* *: p<0.01$ by Tukey's multiple comparisons test following twoway ANOVA. ns: not significant. 


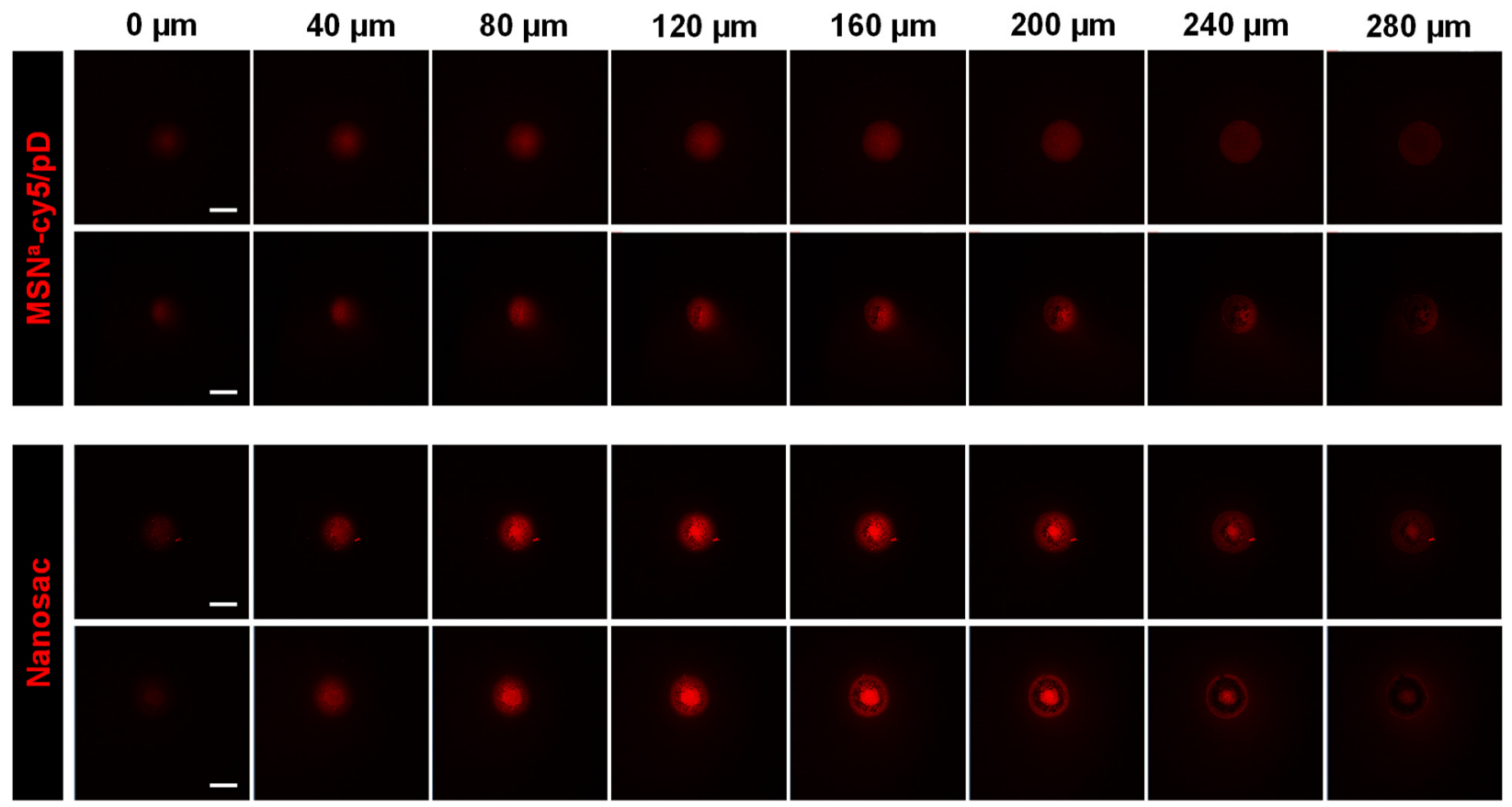

Supporting Fig. 23. Z-section images of CT26 tumor spheroids incubated with $\mathrm{MSN}^{\mathrm{a}}$-cy5/pD or Nanosac. Scale bars: $500 \mu \mathrm{m}$. 


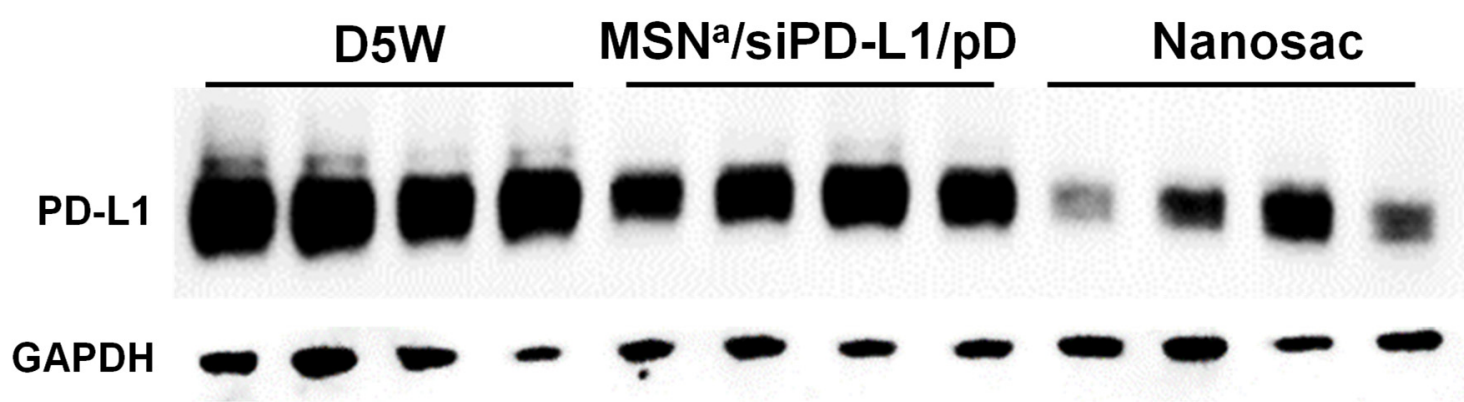

Supporting Fig. 24. Western blot of PD-L1 expression in CT26 tumors of Balb/c mice treated with D5W, MSN $/$ siPD-L1/pD, or Nanosac (siPD-L1: $0.75 \mathrm{mg} / \mathrm{kg} / \mathrm{time}, \mathrm{q} 2 \mathrm{~d} \times 10$ ). $\mathrm{n}=4$ mice per group. 

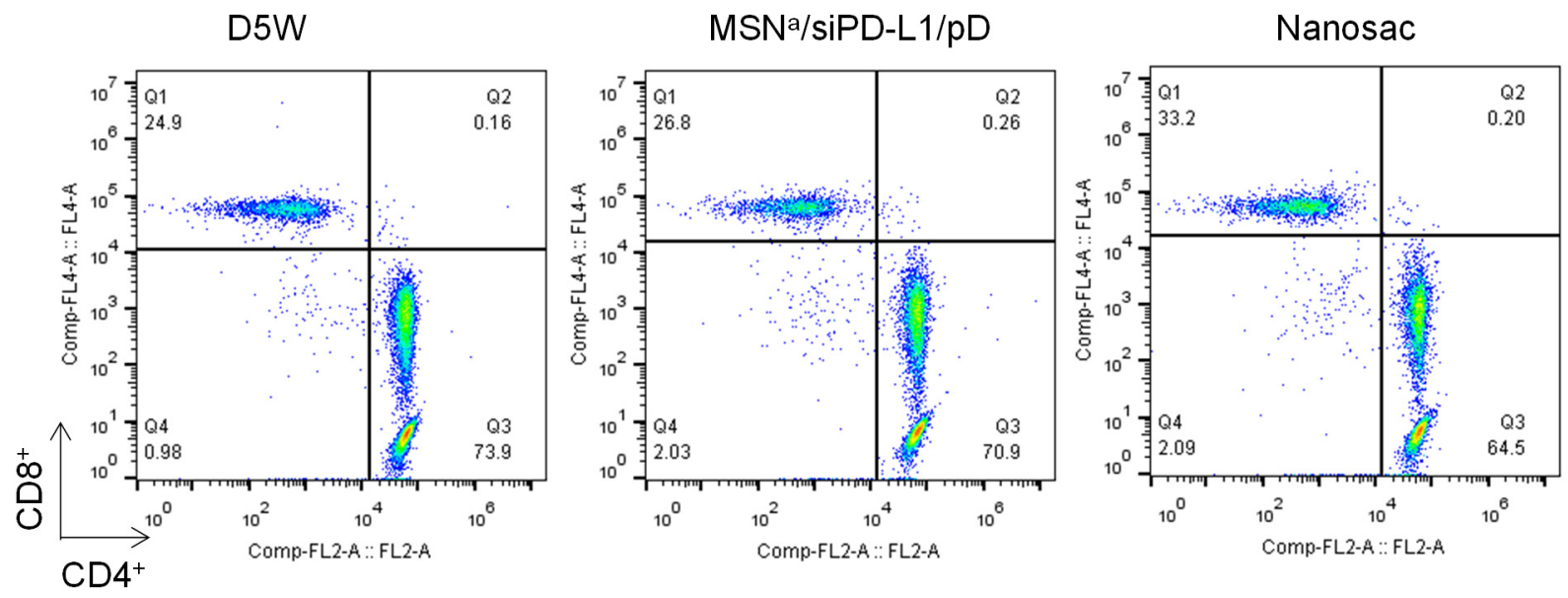

Supporting Fig. 25. Representative flow cytometric dot plots of $\mathrm{CD}^{+}$and $\mathrm{CD} 4^{+}$cells in tumor draining lymph nodes (TDLNs) of treated animals. 


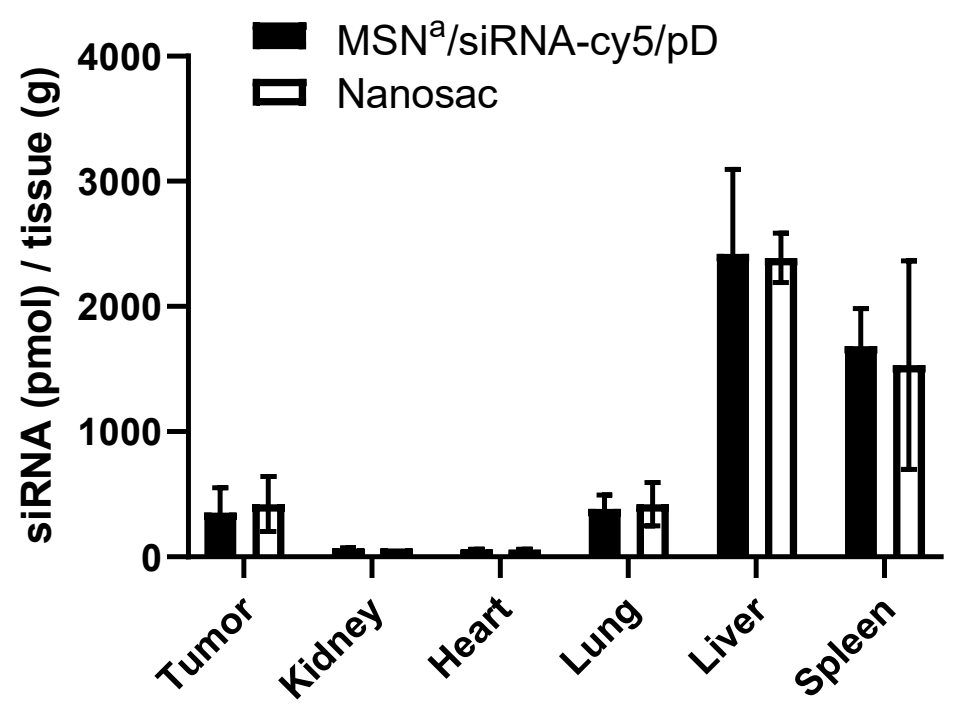

Supporting Fig. 26. Biodistribution of siRNA-cy 5 delivered by MSN $/ \mathrm{siRNA}$-cy $5 / \mathrm{pD}$ and Nanosac (siPD-L1-cy5: $0.75 \mathrm{mg} / \mathrm{kg}$ ) in Balb/c mice bearing CT26 tumors, $24 \mathrm{~h}$ after IV injection. $\mathrm{n}=3$ mice per treatment (mean \pm s.d). 


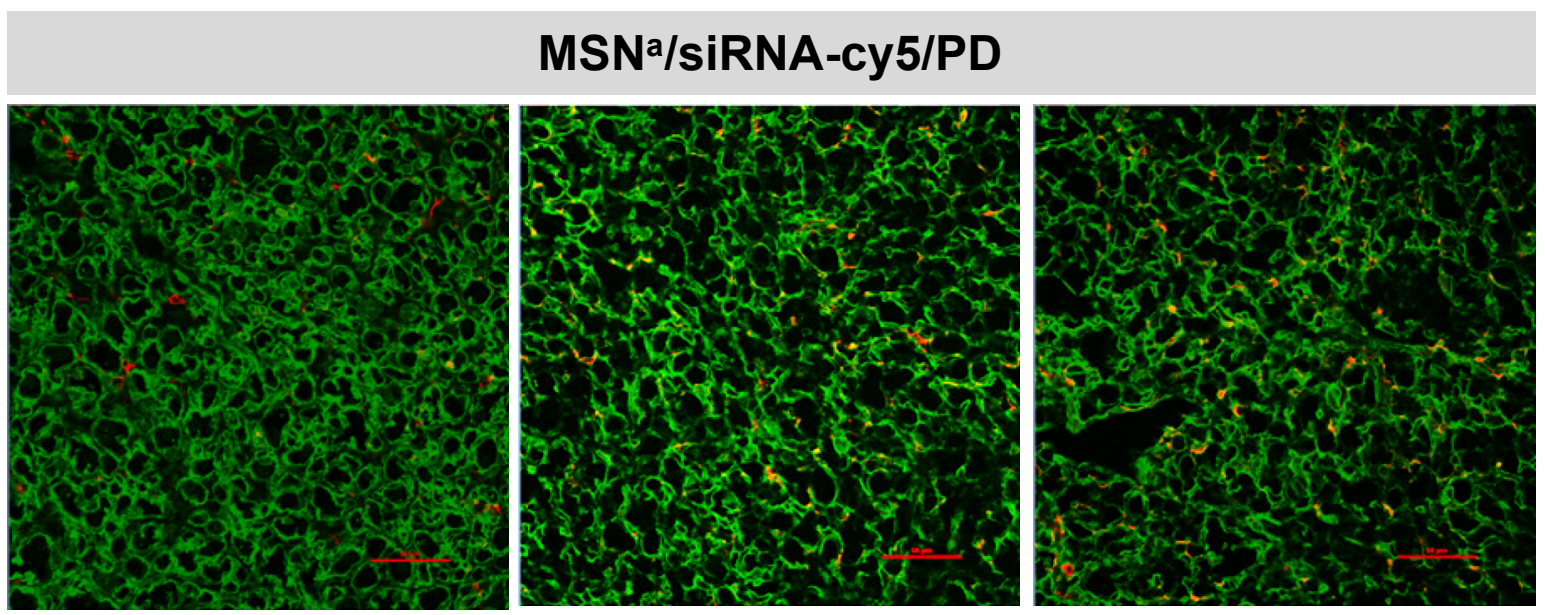

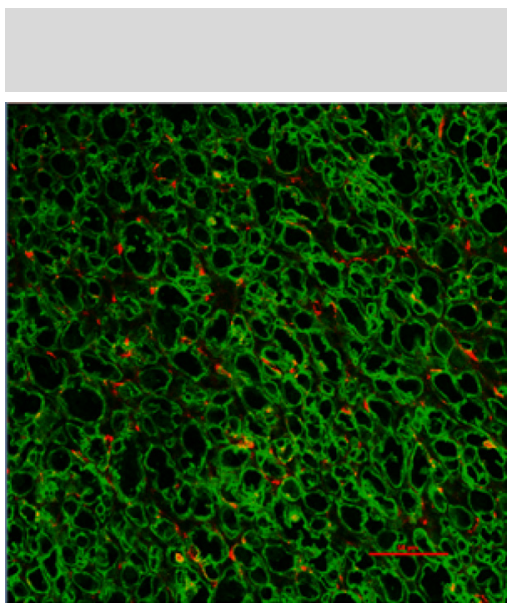

Vessel NP
Nanosac
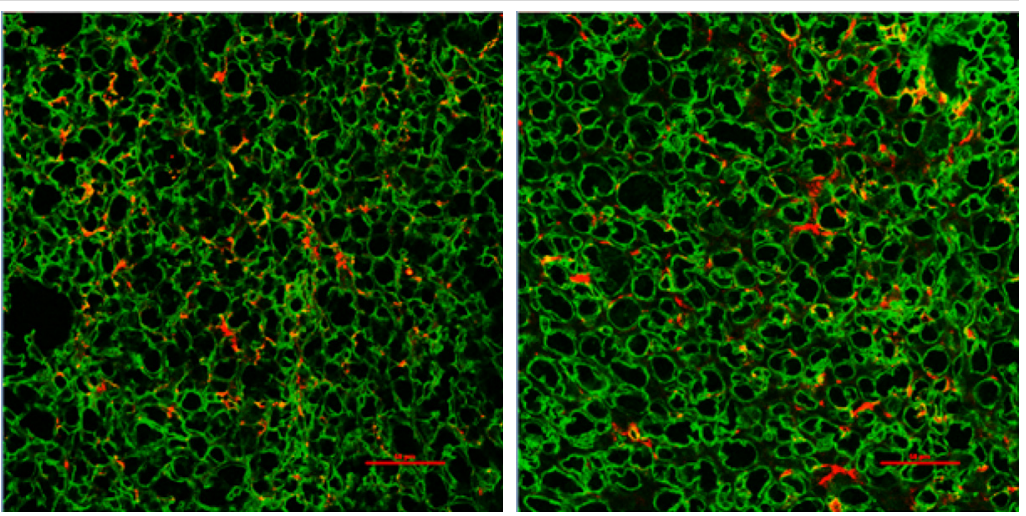

$50 \mu \mathrm{m}$

Supporting Fig. 27. Fluorescence micrographs of tumor sections showing FITC-lectin-stained vessels (green) and MSN $/ \mathrm{siRNA}-\mathrm{cy} 5 / \mathrm{pD}$ or Nanosac (red). Scale bar: $50 \mu \mathrm{m}$. 


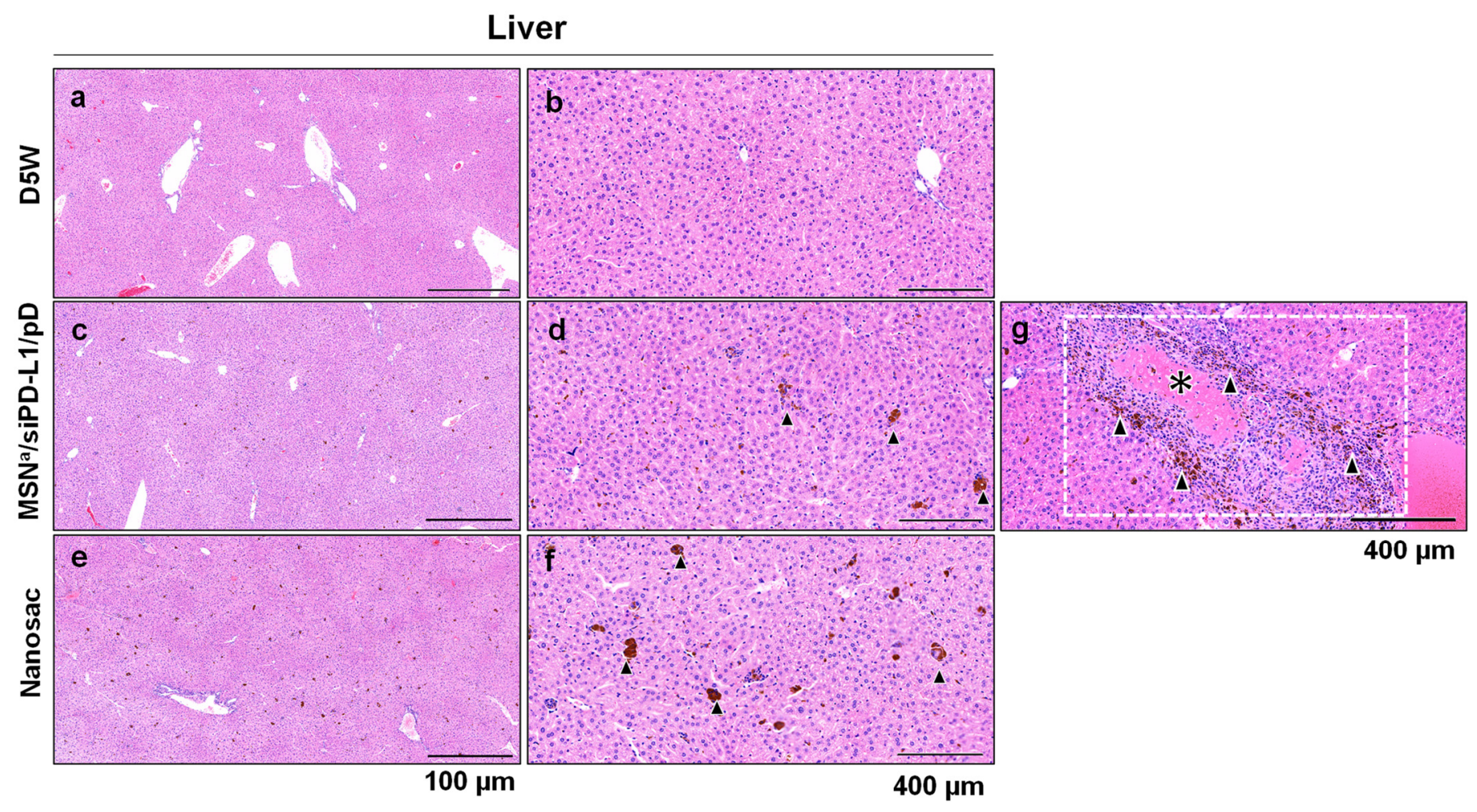

Supporting Fig. 28. Low (a, c, e) and high (b, d, f) magnification photomicrographs of hematoxylin and eosin (H\&E)-stained liver sections. Arrowheads in (d) and (f) indicate clusters of NPs laden in Kupffer cells. (g) In one of the MSN $/$ siPD-L1/pD-treated animals, the liver section showed severe mixed infiltrates (dashed box) associated with abundant clusters of macrophages laden with NPs (arrowheads) in a periportal region with hepatic dropout and necrosis (asterisk). 


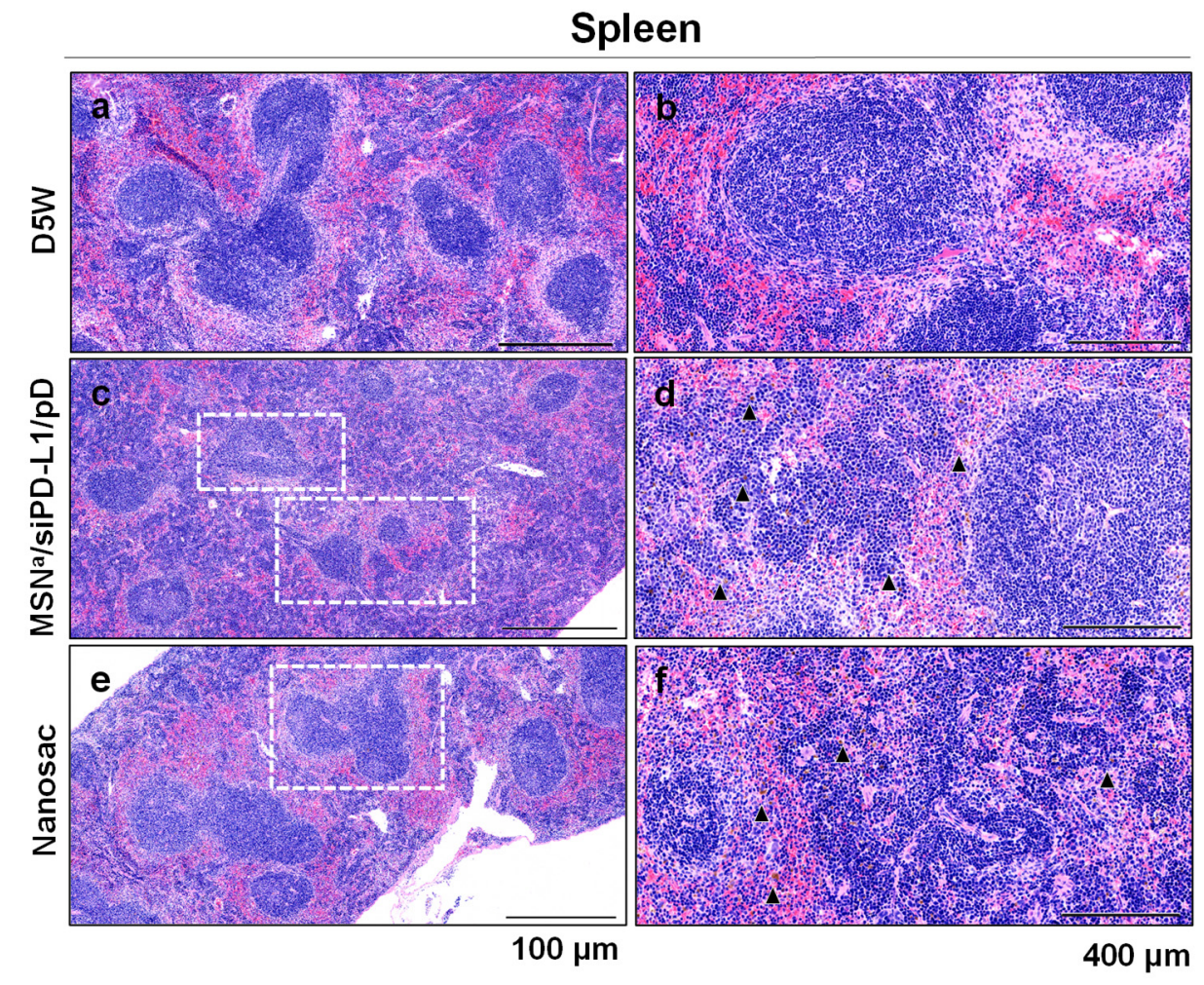

Supporting Fig. 29. Low (a, c, e) and high (b, d, f) magnification photomicrographs of H\&Estained spleen sections. White dashed boxes in (c, e) show a less prominent zonal pattern due to increased macrophages and plasma cells in the white pulp. Arrowheads in (d) and (f) indicate macrophages laden with NPs. 

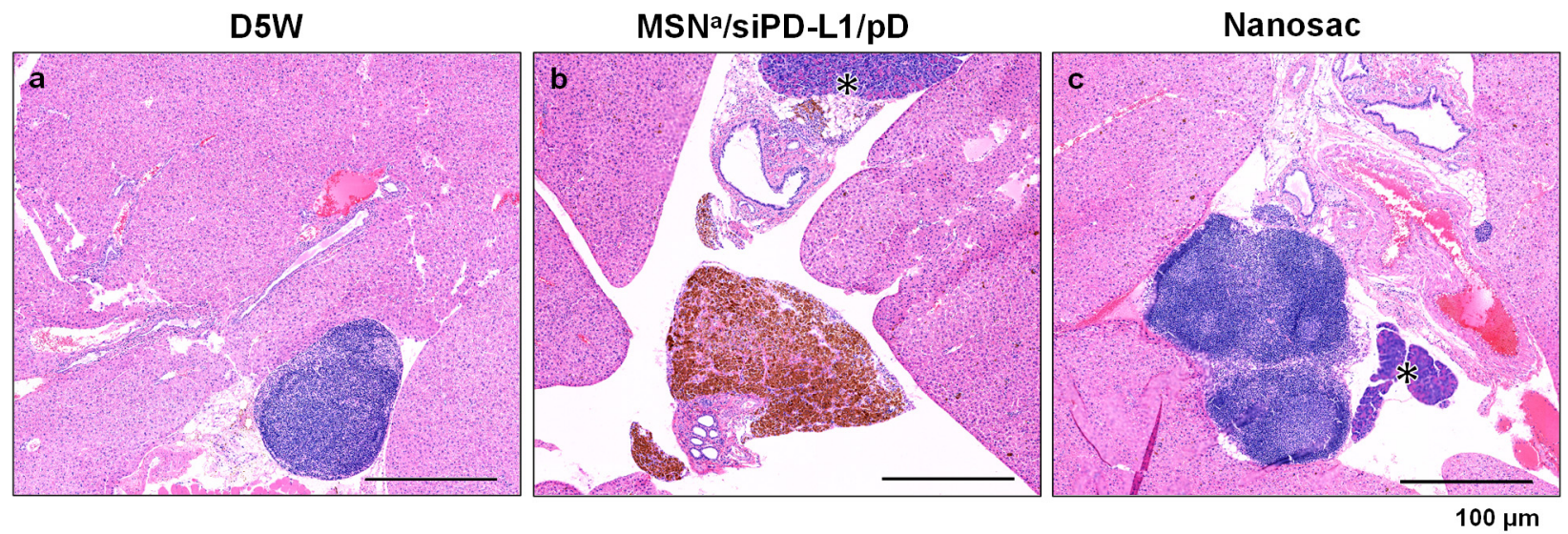

Supporting Fig. 30. Representative H\&E images of peripancreatic (pancreas designated by asterisk) and perihepatic lymph nodes. 

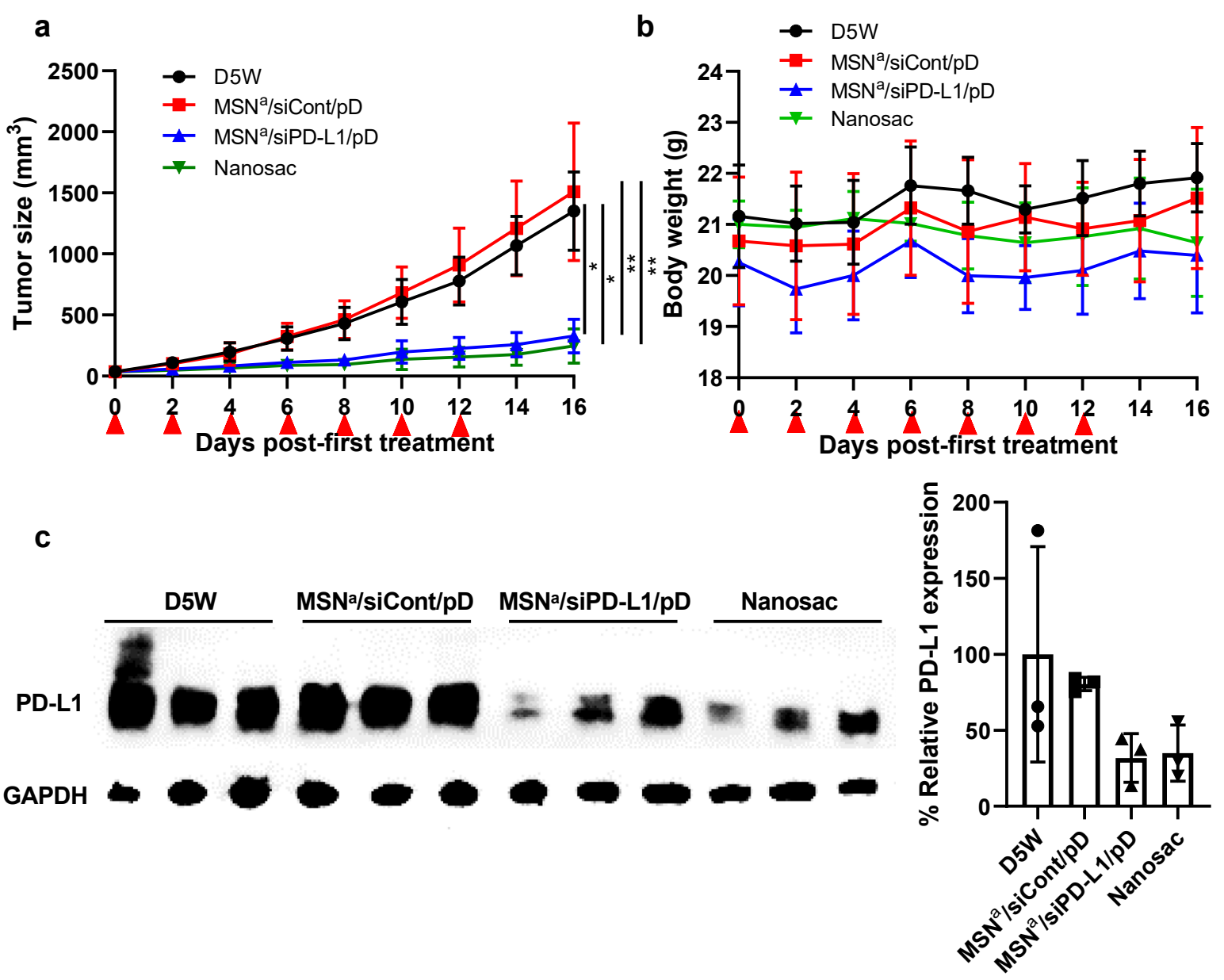

Supporting Fig. 31. Anti-tumor activity of D5W, MSN $/ \mathrm{siCont} / \mathrm{pD}, \mathrm{MSN} / \mathrm{siPD}-\mathrm{L} 1 / \mathrm{pD}$, and Nanosac in Balb/c mice bearing CT26 tumors (siPD-L1: $1.5 \mathrm{mg} / \mathrm{kg} / \mathrm{time}, \mathrm{q} 2 \mathrm{~d} \times 7$ ). (a) Average tumor size $\left(\mathrm{mm}^{3}\right){ }^{*}: \mathrm{p}<0.05 ; *: \mathrm{p}<0.01$ between average tumor sizes on day 16 post-first injection by Tukey's multiple comparisons test following two-way repeated measures ANOVA. (b) Average body weight (g). Arrowheads indicate times of treatment. $\mathrm{n}=5$ mice per treatment (mean \pm s.d). (c) Expression of PD-L1 in CT26 tumors. $n=3$ mice per group (mean \pm s.d). 

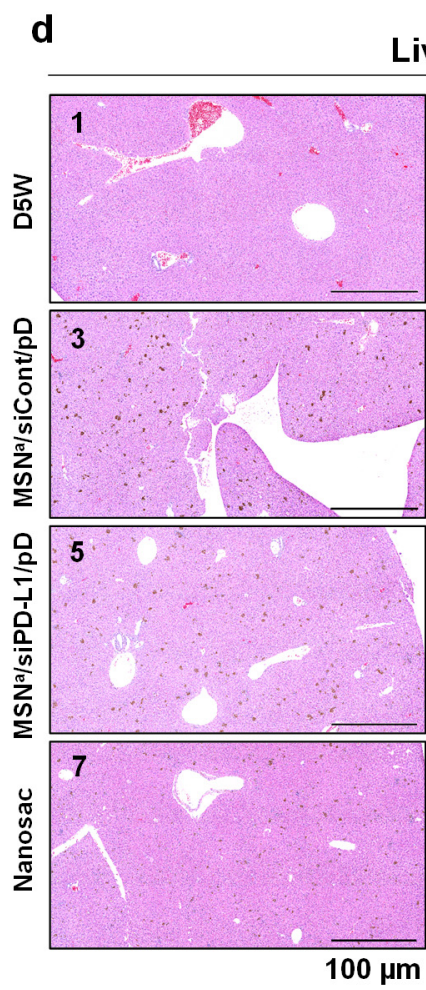

Liver
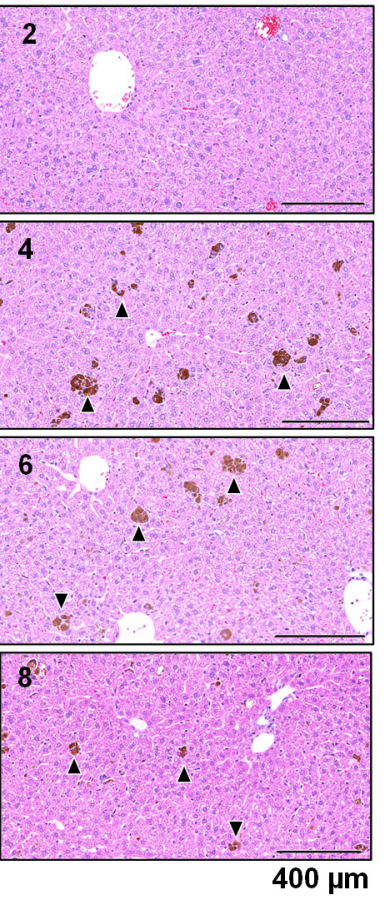

Spleen
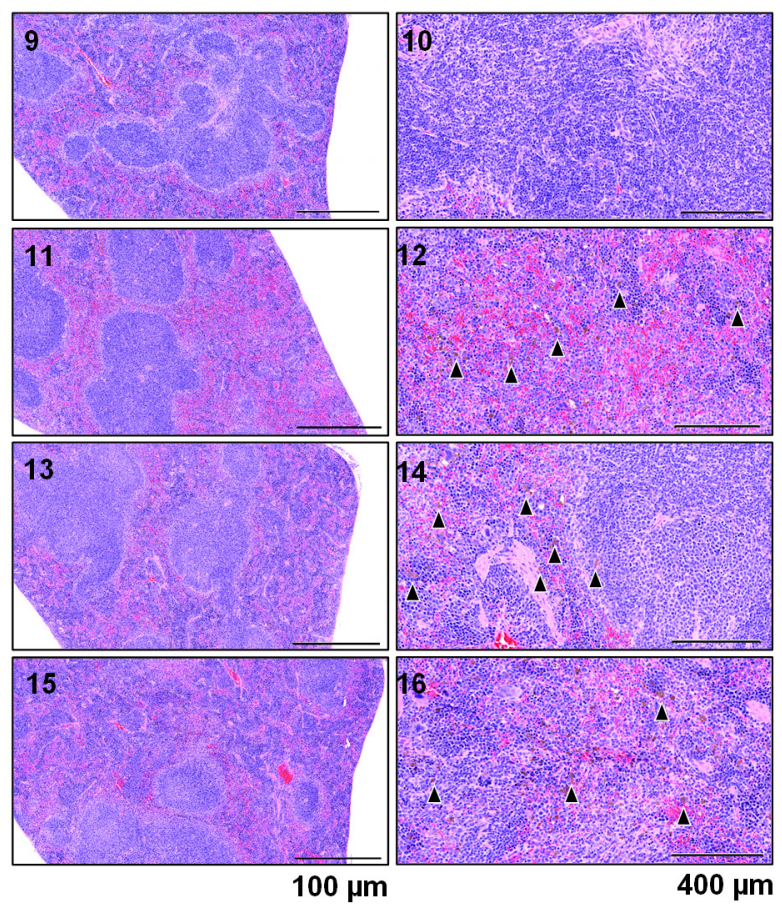

Supporting Fig. 31 (continued). (d) Photomicrographs of H\&E-stained liver and spleen sections. No abnormalities were observed in either organ in all treatment groups. Liver: $\mathrm{MSN}^{\mathrm{a}} / \mathrm{siCont} / \mathrm{pD}$ (3) and MSN/siPD-L1/pD (5) groups have abundant brown dots, which are clusters of NPs laden in Kupffer cells as shown in high magnification photos (arrowheads in 4, 6). Nanosac (7) group also shows brown dots, smaller and fewer than those of $\mathrm{MSN}^{\mathrm{a}} / \mathrm{siCont} / \mathrm{pD}$ (3) and MSN $/$ siPD-L1/pD (5). Nanosac also occupies Kupffer cells but in fewer clusters (arrowheads in 8). Spleen: MSNa/siCont/pD (11), MSNa/siPD-L1/pD (13), and Nanosac (15) groups show mild sinusoidal expansion. On high magnification, sinusoids are expanded by macrophages, often containing brown pigments (NPs) (arrowheads in 12, 14, and 16). 
Supporting mov. 1. Time-lapse intravital microscopic movie of Nanosac circulating in CT26 tumor-bearing BALB/c mice.

Supporting mov. 2. Time-lapse intravital microscopic movie of $\mathrm{MSN}^{\mathrm{a}}$-cy $5 / \mathrm{pD}$ circulating in CT26 tumor-bearing BALB/c mice. 